\title{
Effect of An Educational Intervention About Occupational Safety on Knowledge and Practices of Male Students in Technical Mechanical Secondary Schools in El-Behira Governorate
}

\author{
Shaimaa Mohammed Amin ${ }^{1} \quad$ Mariam haggag soliman $^{2} \quad$ Abeer Abdel Aziz Madian $^{3}$ \\ Amira Abdelraheem Mohamed $\mathrm{Ali}^{4}$ \\ 1.Assistant lecturer at community health nursing, faculty of nursing, Damanhour University \\ 2.Professor of Community Health Nursing, faculty of nursing, Damanhour University \\ 3.Assistant Professor of Community Health Nursing, faculty of nursing, Damanhour University \\ 4.Lecturer of occupational hygiene and air pollution, High Institute of Public Health, Alexandria University
}

\begin{abstract}
Background. Occupational health and safety is one of the most important aspects of human concern. The Aim of this study is to evaluate the effect of educational intervention about occupational safety on knowledge and practice of male students in technical mechanical secondary schools in El-Behira governorate. Hypothesis: Technical Mechanical secondary school male students who engage in occupational safety intervention will demonstrate higher level of knowledge and practice than those who are not . Material and Methods: Quasiexperimental pre/post-test research design. Settings: the study was conducted in four technical mechanical secondary schools in El-Behira governorate. Subjects: the study was carried on 280 students (140 were cases and 140 were control).Data collection tools: data were collected using three tools: structured self-administered questionnaire, Students' knowledge regarding occupational safety and observation check list regarding safety measures practices in the training workshops.Results: conveyed that less than half $(46.4 \%, 47.9 \%)$ of both the study and the control group respectively were aged between 16 to less than 17 years. All the study group and the control one had poor knowledge regarding occupational health and safety before conduction of the educational intervention, whereas after implementation of the educational intervention, more than three quarters of the study group (85.7\%) had good knowledge whereas the vast majority of the control group (99.3\%) still had poor knowledge . There was a statistically significant relation between the department and the total knowledge score of the study group $\left(\mathrm{X}^{2}=14.150, \mathrm{P}=0.007^{*}\right)$.Conclusion: The educational program is successful in attaining its aim of positively improving knowledge, and practice of the study group about Occupational Health and Safety.Recommendations: this study recommend the necessity of provision of personal protective equipment to the students in the school workshops and integrating occupational safety topics in their curriculum. Keywords: Educational intervention, occupational health, Knowledge, Technical secondary schools.
\end{abstract}

DOI: $10.7176 / \mathrm{JHMN} / 80-05$

Publication date:September $30^{\text {th }} 2020$

\section{Introduction}

Young people spend an expansive extent of their day in school or seeking after school-related exercises. Whereas, the essential reason of school is the scholastic advancement of students, its impacts on youths are distant broader. Moreover, enveloping their physical and mental wellbeing, security, civic engagement, and social $^{(1)}$.development (WHO 2018). A secure environment is a prerequisite for successful learning, so there must be school programs in place to diminish levels of injuries as part of its wider plan to improve academic performance ${ }^{(2)}$.

Technical education is the cornerstone for all advancement efforts at any society. It plays a critical part in pushing up the advancement wheel and in accomplishing its maximum rates ${ }^{(3)}$. Whatever the improvement plans quality is, they couldn't accomplish their goals and their targeted rates without the existence of scientifically and technically qualified human cadres in all production fields ${ }^{(4)}$.

Vocational Secondary School is one of the branches in which the students will acquire the potential of technical talent from the technical field. To produce the potential of this professional future, specialized training is essential. In producing students who are exceedingly talented, they ordinarily utilize the practical training workshop more frequent for their technical practices. This is to reinforce the knowledge gained by students before they enter the real working environment ${ }^{(5)}$.

A secure school is one where the entire school environment enables students, teachers, administrators, and staff to communicate in a favorable, non-threatening way that reflects the educational mission of school while fostering positive relationship and personal development ${ }^{(6)}$.Safety at school includes identifying and implementing suitable policies and programs to ensure the health of students and staff ${ }^{(7)}$.

Creating a healthy school environment requires the inclusion of virtually everyone in the school students, administrators, teachers, guardians, school counselors, and nurses .Hence safe and supportive schools refers to 
the provision of an environment that protects the emotional, psychological and physical prosperity of students ${ }^{(8)}$

Students in technical school face risks at their work stations in schools and furthermore at their work such as physical, chemical and psychosocial hazards. Since the scheme of the technological and vocational education is career oriented, the mentality of the students usually emphasizes specialized subjects and overlooks the overall curricula $^{(9)}$.

Occupational health and safety (OHS) training is a principal component in work environment risk control programs. Various safety and health standards for hazard control contain requirements for training pointed at diminishing risk factors for injury, disease or death. Combined with management responsibility, which is fundamental, training is a vital portion of a comprehensive risk control program. Improving the effectiveness of OHS training efforts and other interventions is vital particularly as workplaces and workforces change ${ }^{(10)}$.

Unintentional injuries are harmful acts that occurred without any intention of causing damage to oneself or others. Unintentional accidents amongst teenagers and young adults age 21 and younger, an already susceptible, vulnerable subpopulation, are ongoing public health issue ${ }^{(11)}$. Nearly 18.1 million young workers under age 24 comprised about $13 \%$ of the workforce in the U.S. in 2013. In 2017, 375 young U.S. workers died from workrelated injuries. Several researches published that there have been high occurrence of injuries amongst young people due to knowledge deficit about occupational fitness and safety and also lack of training about occupational dangers ${ }^{(12)}$.

Occupational health and protection is the concern of human wellbeing. Nowadays industrialization and service giving sectors development is accelerating resulting in enormous occupational health problems. Occupational safety and health hazards currently regarded as a driving force toward finding solutions how to prevent it from the manufacturing industries worker bad consequence ${ }^{(13)}$. In latest years, the quality, health, knowledge and protection necessities in several countries have been greater stringent than was the case seen before. Several studies concluded that pressures from communities have led to the enactment of a range of safety legislations and safety standards in different regions for several industries ${ }^{(14)}$.

A study carried out in United States (2018) revealed that extra than half $(57 \%)$ of the study group of students had experienced injury during training in the mechanical workshops. The most commonly injured body parts had been hands, fingers, and legs .The most commonly reported injury kind was cuts/lacerations. Moreover, the study revealed that these injuries have been due to lack of knowledge about occupational fitness and safety (15)

A study conducted in USA 2018 illustrated that more than three quarters (77\%) of technical education students have poor knowledge about occupational fitness. More over the study illustrated that the majority of the students didn't wear personal protective equipment's during training in the workshop and therefore about $30 \%$ of them had experienced injuries during training ${ }^{(16)}$.

The Occupational health nurse $(\mathrm{OHN})$ performs an important role in assisting to defend the fitness of workers. The role of $\mathrm{OHN}$ is to authorize workers to make informed health decisions while also overseeing the fitness dangers and costs linked with the employment relations between employees and the enterprise ${ }^{(17)}$. The main functions of the occupational health nurse are to keep employees healthy, prevent illness and ensuring a safe working environment. The occupational health nurse is in an ideal situation to provide guidance, counseling, and coaching for employees who choose to enhance their health ${ }^{(18)}$.

\section{Significance of the study}

Students in technical school face risks at their work stations in schools and furthermore at their work such as physical, chemical and psychosocial hazards. Since the scheme of the technological and vocational education is career oriented, the mentality of the students usually emphasizes specialized subjects and overlooks the overall curricula $^{(9)}$.

A study conducted in Ethiopia (2019) revealed that more than two-thirds $(48.1 \%)$ of the welders never heard about occupational hazards. With respect to fire hazards more than half (56\%) of the study subjects didn't have knowledge about fire hazards, whereas more than three fourth $(78 \%)$ of the respondents didn't have knowledge about electrical hazards. However, less than one third $(28 \%)$ of the study subjects didn't have knowledge about accident prevention ${ }^{(19)}$.

A study conducted in Sweden (2014) concluded that vocational education pupils had poor knowledge regarding occupational risks and protective measures. Post program implementation about occupational safety, the majority $(95 \%)$ of the students had good knowledge concerning occupational health and personal protective equipment ${ }^{(20)}$.

Although some studies are done on the prevalence of injuries among technical education students, information on occupational health and safety among technical mechanical secondary school students is still minimal. Furthermore, there are limited studies that specifically determine effect of educational intervention about occupational safety on knowledge and practice of male students in technical mechanical secondary schools. Therefore, the intent of the present study was to evaluate the effect of an educational intervention about 
occupational safety on knowledge and practice of male students in technical mechanical secondary schools in ElBehira Governorate in order to improve their knowledge, practice toward occupational safety, hence improve their health.

\section{Aim of the study}

Aim of the study is to:

- Evaluate the effect of educational intervention about occupational safety on knowledge and practice of male students in technical mechanical secondary schools in El-Behira governorate

\section{Research hypothesis}

- Technical Mechanical secondary school male students who engage in occupational safety intervention will demonstrate higher level of knowledge and practice than those who are not

\section{Material and Methods}

\subsection{Material:}

\subsubsection{Research design :}

Quasi experimental (pre-posttest) study design was carried out to demeanor this study.

\subsubsection{Study setting :}

The study was conducted in Four schools out of 13 schools (around one quarter) harvesting the highest numbers of students at the second level namely; Damnhour Technical Mechanical Secondary School, Kafr-El-Dawar Technical Mechanical Secondary School, Abo Homos Technical Mechanical Secondary School, and Hosh Eisa Technical Mechanical Secondary School

\subsubsection{Subjects:}

The target population of this study was the male students in the technical mechanical secondary schools who meet the following inclusion criteria:

- Enrolled in the second academic year

- Free from chronic disease

- Free from disability

\subsubsection{Sampling technique:}

By using multi stage sampling technique, the following steps were done:

- El-Behira governorate was classified into 18 educational directorates. only 13 directorate have technical mechanical secondary schools

- Four schools (constituted 25\%) having the highest numbers of students were purposively selected .

- From each school, the students enrolled in the second academic year were included in the study using systematic random sampling technique to select the required sample size of 280 students.

- The study sample was randomly assigned (lottery) to one of two groups, either the experimental group ( $n=140$ students) or control group $(n=140)$ students .The experimental group received the occupational safety program, while the control group received the routine instructions from their teachers.

\subsubsection{Sample size:}

The sample size was calculated by using EPI info7software based on the total population of 1104 and an expected frequency of $50 \%$ of hazards with margin error of $5 \%$ and confidence interval of $95 \%$. This resulted in minimum required sample size of 280 students. 140 students were selected as an experimental group and 140 students were randomly selected as control group from each of the previously selected settings according to the following table.

Number of Students Selected From Different Departments

\begin{tabular}{|c|c|c|c|c|c|c|c|}
\hline \multirow{3}{*}{ School } & \multicolumn{6}{|c|}{ Department } & \multirow[t]{3}{*}{ Total } \\
\hline & \multicolumn{2}{|c|}{ Welding } & \multicolumn{2}{|c|}{ Lathing } & \multicolumn{2}{|c|}{ Foundry } & \\
\hline & $\begin{array}{l}\tilde{E} \\
\tilde{E} \\
\tilde{U}\end{array}$ & 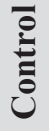 & $\begin{array}{l}\tilde{E} \\
\tilde{E} \\
\tilde{E}\end{array}$ & نे & $\begin{array}{l}\tilde{E} \\
\tilde{E} \\
\tilde{U}\end{array}$ & ن를 & \\
\hline Damnhour Technical Mechanical Secondary School & 13 & 13 & 13 & 13 & 12 & 12 & 76 \\
\hline Abo Homos Technical Mechanical Secondary School & & & 34 & 35 & & & 69 \\
\hline Kafr-El-Dawar Technical Mechanical Secondary School & 19 & 19 & 19 & 19 & & & 76 \\
\hline Hosh Eisa Technical Mechanical Secondary School & 10 & 10 & 10 & 10 & 10 & 9 & 59 \\
\hline
\end{tabular}

2.1.6 Tools for data collection:

Data were collected using the following three tools. 
Tool (I): structured self-administered questionnaire:

It was developed by the researcher and is composed of three parts. The first part is developed to collect data related to socio-demographic characteristics of the students: such as age, Academic department, residence, crowding index, family income, father\& mother education and occupation. The second part included assessment of student's general health status like presence of health problems in the last 6 months, medication used, lab investigation done before, and presence of vision or hearing problems. The third part involved questions related to lifestyle of the students as physical exercises, sleeping patterns, eating habits, risk taking behaviors such as smoking, alcohol consumption, and drug abuse.

Tool (II): Students' knowledge regarding occupational safety

It was concerned with the following items:

- Occupational safety in schools (definition, occupational safety committee in schools, activities of occupational safety committee )

- Possible environmental hazards present in school from their point of view

- Accidents hazards and their prevention

- Safety measures needed and adopted to overcome these hazards

- Personal protective equipment, and importance of using it

- $\quad$ First aid measures

- Fire hazards and their prevention

Scoring system:

The students' knowledge about safety measures were calculated for each item. It consists of 62 items. A score of (2) will be given to the complete and correct answer, a score of (1) for correct but incomplete answer and a score of (0) for the wrong or missed answer. The total knowledge score was calculated and ranged from (0-124) which further categorized into three levels as follows:

\begin{tabular}{|l|l|}
\hline \multicolumn{1}{|c|}{ Classification } & \multicolumn{1}{c|}{ Score } \\
\hline Good & $75 \%-100 \%(93-124$ points $)$ \\
\hline Fair & $<75 \%-50 \%(62-<93$ points $)$ \\
\hline Poor & $<50 \%(<62$ points $)$ \\
\hline
\end{tabular}

Tool (III): Observation check list regarding safety measures practices in the training workshops

An observational checklist was developed by the researcher after reading the recent literature in order to assess safety measures utilized in the mechanical workshops. It included the following parts:

Part I: Workshops Environmental safety measures

This part included data as: housekeeping, ventilation, noise control, lighting system, fire protection, electrical safety and workshop design

Part II: Work process safety measures

This part included safety measures adopted in various departments like

Welding, lathing, and foundry.

Part III: Protective devices used by students:

This part included different personal protective equipment utilized by students in different departments of workshops such as safety glasses, mask, ear plug, gloves, apron, safety shoes...etc

Scoring system:

Safety measures adopted in training workshops composed of 75 items and was calculated for each one. Every item was scored (1) if present and (zero) if not present. The total observational score was calculated and ranged between 0-100 and categorized into two levels as follows:

- $75 \%$ or more items of safety measures was considered as having complete safety measures $(\geq \mathbf{7 5 - 1 0 0}$ points ).

- Less than $75 \%$ items of safety measures was considered as having incomplete safety measures. $\quad(<\mathbf{7 5}$ points) .

Tool Content Validity and Reliability:

Tools of the study were examined for content validity by a jury of five experts in the field of community health nursing from the Faculty of Nursing (Damnhour and Alexandria university) and the necessary modifications were done based on their recommendations . The reliability was assured by utilizing test-retest, and the result was 0.9 .

\subsection{Methods:}

- Permission to conduct the study in the selected setting was obtained after explanation of the aim of the study

- Tool I structured self-administered questionnaire: It was developed by the researcher after reviewing of the recent literatures to assess socio-demographic characteristics and life style of the students. Tool II Students' knowledge regarding occupational safety. It was developed by the researcher after reviewing the recent 
literature to assess students' knowledge regarding occupational safety. Tool III Observation check list regarding safety measures practices in the training workshops. Was constructed by the researcher after reviewing the recent literature so as to assess safety measures used in the mechanical workshops. Tools validity, all the study tools (I,II,III) were examined for content validity by a jury of five experts in the field of community health nursing from the Faculty of Nursing (Damnhour and Alexandria university) and the necessary modifications were done based on their recommendations . Tool reliability, the reliability of tool III was conducted using test-retest and the result was 0.9 and the tool seems to be reliable.

- Pilot study: Twenty-eight (10\% of the estimated sample) were piloted to ascertain the clarity, applicability and feasibility of the tools and to detect the obstacles that might impede the data collection process (not included in the original sample ) consequently, all needed modifications were done.

- Data collection phase: The overall data collection process took about seven months (from September 2019 to March 2020).

\section{- Educational intervention:}

The study was implemented through the following three phases: Preparation of the educational intervention, implementation of the educational intervention and evaluation of the educational intervention. Collection of the data covered a period of six months from September 2019 to February 2020.

\section{First Phase: preparation of the educational intervention:}

\section{Assessment phase}

- The researcher visit the study setting and explain the questionnaire to both the study and control group.

- Pre intervention assessment for the students' knowledge and practice about occupational safety was done by the researcher after distributed tool II to be completed by both groups as pretest and to detect their learning needs in the first week before starting the program in each school.

- The observational assessment was calculated by the researcher for both study and control groups to assess safety measures practices in the training workshops by using tool III.

\section{Planning phase}

The intervention of occupational safety was developed by the researcher for the study group according to student's needs assessment and review of literature as to the following steps:

\section{a)Setting the program objectives}

$>$ General objective

To improve the knowledge and practices of male students regarding occupational safety

$>$ Specific objectives

By the end of the educational intervention, the students were able to:

- Define occupational safety

- List activities of occupational safety

- Mention the possible environmental hazards present in their schools

- Mention prevention of accidents and hazards

- Apply safety measures needed to overcome the occupational hazards

- Apply personal protective equipment and mention its importance

- List first aid measures

- Apply prevention of fire hazards

b)Preparation of the content

- The content of the educational intervention of occupational safety was designed to by the researcher to cover all the predetermined objectives .it was developed based on review of relevant recent literature, results of preassessment as well as characteristics of students.

- The content was comprised and formulated as regard to numbers of sessions and teaching methods

- Different educational strategies were utilized as lectures, brain storming, group discussion, and role play

- Different teaching aids were used to facilitate and illustrate teaching such as posters, handout, slide data show and videos.

\section{Second Phase: Implementation of the Educational Intervention}

1) This phase included the implementation of the planned educational intervention.

2)The students were divided into groups at each school, each group was contain around (15-20) students.

3) At the beginning of the first session, students were oriented to the aim of the study, phases and the educational intervention sessions (time, duration, and contents). The researcher focused on the importance of continuous attendance and active participation. In each session students questions were answered, then handout were distributed to them.

4)Timetable of the educational intervention regarding occupational health and safety 


\begin{tabular}{|c|c|c|c|c|c|}
\hline Days & Contents & $\begin{array}{l}\text { Teaching } \\
\text { methods }\end{array}$ & Media & Time & Evaluation \\
\hline $\begin{array}{l}\text { First } \\
\text { day } \\
\text { Session } \\
1\end{array}$ & $\begin{array}{l}>\text { Orientation of the } \\
\text { participants } \\
\text { Explain the purpose and } \\
\text { nature of the study for them } \\
>\quad \text { Introduction (Technical } \\
\text { education in Egypt and its } \\
\text { importance) } \\
>\text { Overview about school } \\
\text { safety ( definition of school } \\
\text { safety, type of hazards in } \\
\text { school, safety precautions in } \\
\text { school, the role of school } \\
\text { safety committee) }\end{array}$ & $\begin{array}{ll}\text { - } & \text { Discussion } \\
\text { - } & \text { Lecture } \\
\text { - } & \text { Brain } \\
& \text { storming }\end{array}$ & $\begin{array}{ll}\text { - } & \text { Brochure } \\
\text { - } & \text { Data show }\end{array}$ & $\begin{array}{l}1 \\
\text { hour }\end{array}$ & $\begin{array}{ll}> & \text { Pretest } \\
> & \text { Post test }\end{array}$ \\
\hline $\begin{array}{l}\text { Second } \\
\quad \text { day } \\
\text { Session } \\
2\end{array}$ & $\begin{array}{l}\text { Occupational health } \\
\text { (definition of occupational } \\
\text { health, objectives of } \\
\text { occupational health } \\
\text { occupational hazard } \\
\text { meaning, various sorts of } \\
\text { occupational risks) }\end{array}$ & $\begin{array}{ll}\text { - } & \text { Discussion } \\
\text { - } & \text { Lecture } \\
\text { - } & \text { Brain } \\
& \text { storming }\end{array}$ & $\begin{array}{ll}\text { - } & \text { Data show } \\
\text { - } & \text { Brochure } \\
\text { - } & \text { Poster }\end{array}$ & $\begin{array}{l}1 \\
\text { hour }\end{array}$ & $\begin{array}{ll}> & \text { Pretest } \\
> & \text { Post test }\end{array}$ \\
\hline $\begin{array}{l}\text { Third } \\
\text { day } \\
\text { Session } \\
3\end{array}$ & $\begin{array}{l}\text { D Safety precautions in } \\
\text { mechanical workshops } \\
\text { (occupational hazards and } \\
\text { protective measures in } \\
\text { welding ,lathing and } \\
\text { foundry workshops } \\
\text { identification of safety } \\
\text { precautions that must be } \\
\text { taken during working in the } \\
\text { workshop) }\end{array}$ & $\begin{array}{ll}\text { - } & \text { Discussion } \\
\text { - } & \text { Lecture } \\
\text { - } & \text { Brain } \\
& \text { storming }\end{array}$ & $\begin{array}{ll}- & \text { Data show } \\
\text { - } & \text { Brochure } \\
\text { - } & \text { Video about } \\
& \text { occupationa } \\
& 1 \text { hazards }\end{array}$ & $\begin{array}{l}1 \\
\text { hour }\end{array}$ & $\begin{array}{ll}> & \text { Pretest } \\
> & \text { Post test }\end{array}$ \\
\hline $\begin{array}{l}\text { Fourth } \\
\text { day } \\
\text { Session } \\
\quad 4\end{array}$ & $\begin{array}{l}\text { Personal protective equipment } \\
\text { ( definition, importance and } \\
\text { different types of personal } \\
\text { protective equipment) }\end{array}$ & $\begin{array}{ll}\text { - } & \text { Discussion } \\
\text { - } & \text { Lecture } \\
\text { - } & \text { Brain } \\
& \text { storming }\end{array}$ & $\begin{array}{ll}\text { - } & \text { Data show } \\
\text { - } & \text { Brochure } \\
\text { - } & \text { Video about } \\
\text { personal } \\
\text { protective } \\
\text { equipment } \\
\end{array}$ & $\begin{array}{l}1 \\
\text { hour }\end{array}$ & $\begin{array}{ll}> & \text { Pretest } \\
> & \text { Post test }\end{array}$ \\
\hline $\begin{array}{l}\text { Fifth } \\
\text { day } \\
\text { Session } \\
5\end{array}$ & $\begin{array}{l}>\text { First aid ( definition, first aid } \\
\text { box contents, principles of } \\
\text { first aid, priorities of first } \\
\text { aid, first aid survey } \\
>\text { Heart attack first aid } \\
\text { measures } \\
>\text { definition, signs and } \\
\text { symptoms, application of } \\
\text { first aid ) } \\
>\text { Burn ( definition, causes of } \\
\text { burn, degrees of burn, } \\
\text { application o first aid) }\end{array}$ & $\begin{array}{ll}\text { - } & \text { Group } \\
& \text { discussion } \\
\text { - } & \text { Lecture } \\
\text { - } & \text { Brain } \\
& \text { storming } \\
\text { - } & \text { Role play } \\
\text { - } & \text { Demonstration } \\
& \text { and } \\
\text { re- } \\
\text { demonstration }\end{array}$ & $\begin{array}{ll}\text { - } & \text { Data show } \\
\text { - } & \text { Brochure } \\
\text { - } & \text { Video about } \\
\text { heart attack } \\
\text { first aid } \\
\text { - } \quad \text { Video about } \\
\text { burn first } \\
\text { aid }\end{array}$ & $\begin{array}{l}1 \\
\text { hour }\end{array}$ & $\begin{array}{ll}> & \text { Pretest } \\
> & \text { Post test }\end{array}$ \\
\hline
\end{tabular}




\begin{tabular}{|c|c|c|c|c|c|}
\hline Days & Contents & $\begin{array}{l}\text { Teaching } \\
\text { methods }\end{array}$ & Media & Time & Evaluation \\
\hline $\begin{array}{l}\text { Sixth } \\
\text { day } \\
\text { Session } \\
6\end{array}$ & 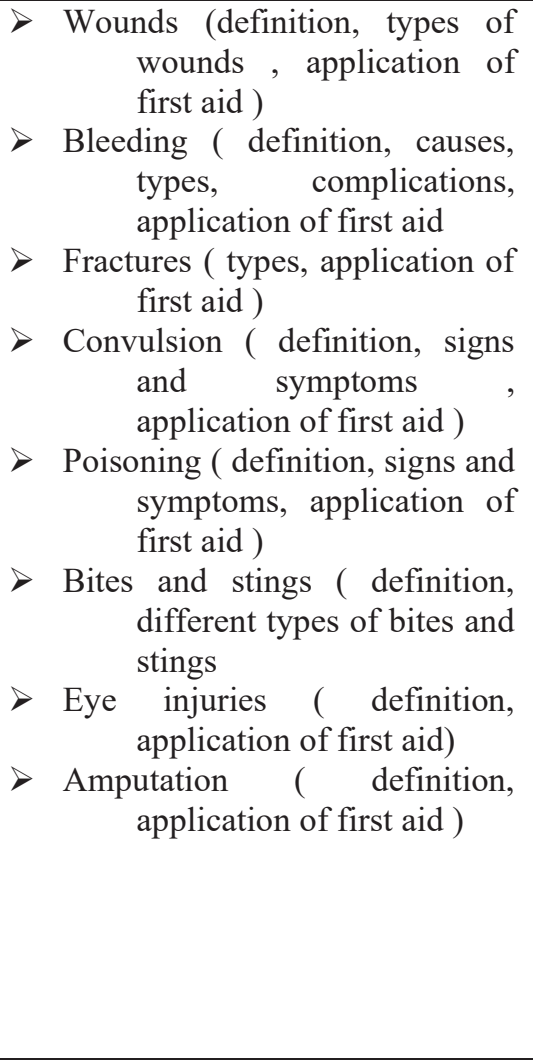 & $\begin{array}{l}\text { - } \text { Discussion } \\
\text { - } \quad \text { Lecture } \\
\text { - } \quad \text { Brain storming } \\
\text { - } \quad \text { Role play } \\
\text { - } \quad \text { Demonstration } \\
\text { and } \\
\text { re-demonstration }\end{array}$ & $\begin{array}{ll}\text { - } & \text { Data show } \\
\text { - } & \text { Brochure } \\
\text { - } & \text { Video } \\
\text { about } & \text { wound first } \\
\text { aid } & \text { Video } \\
\text { about } \\
\text { bleeding } \\
\text { first aid } \\
\text { Video } \\
\text { about } \\
\text { fracture } \\
\text { first aid } \\
\text { Video } \\
\text { about } \\
\text { Convulsion } \\
\text { first aid } \\
\text { Video } \\
\text { about eye } \\
\text { injuries first } \\
\text { aid } \\
\text { Video } \\
\text { about } \\
\text { poisoning } \\
\text { first aid } \\
\end{array}$ & 2hours & $\begin{array}{ll}> & \text { Pretest } \\
> & \text { Post } \\
\text { test }\end{array}$ \\
\hline $\begin{array}{c}\text { Seventh } \\
\text { day } \\
\text { Session } \\
7\end{array}$ & $\begin{array}{l}\text { Emergency evacuation drill } \\
\text { ( definition, objectives, duties of } \\
\text { emergency team, measures that } \\
\text { must be available in school, } \\
\text { duties of firefighting teams in } \\
\text { schools, evacuation exercises in } \\
\text { school ) }\end{array}$ & $\begin{array}{ll}\text { - } & \text { Discussion } \\
\text { - } & \text { Lecture } \\
\text { - } & \text { Brain storming } \\
\text { - } & \text { Role play } \\
\text { - } & \text { Demonstration } \\
& \text { And } \\
& \text { re-demonstration } \\
\end{array}$ & $\begin{array}{ll}- & \text { Data show } \\
\text { - } & \text { Brochure } \\
\text { - } & \text { Video about } \\
\text { emergency } \\
\text { evacuation } \\
\text { drill for fire }\end{array}$ & 1hour & $\begin{array}{l}> \\
>\end{array} \begin{array}{l}\text { Pretest } \\
\text { test }\end{array}$ \\
\hline $\begin{array}{c}\text { Eighth } \\
\text { day } \\
\text { Session } \\
8\end{array}$ & $\begin{array}{l}\text { Types of common accidents in } \\
\text { mechanical workshops and } \\
\text { protective measures (definition of } \\
\text { accident, different types of } \\
\text { accidents inside the mechanical } \\
\text { workshops, causes of accidents, } \\
\text { and protective measures }\end{array}$ & $\begin{array}{ll}\text { - } & \text { Discussion } \\
\text { - } & \text { Lecture } \\
\text { - } & \text { Brain storming } \\
\text { - } & \text { Role play } \\
\text { - } & \text { Demonstration } \\
\text { and } \\
\text { re-demonstration }\end{array}$ & $\begin{array}{ll}\text { - } & \text { Data show } \\
\text { - } & \text { Brochure } \\
\text { - } & \text { Poster }\end{array}$ & 1hour & $\begin{array}{ll}> & \text { Pretest } \\
> & \text { Post } \\
& \text { test }\end{array}$ \\
\hline $\begin{array}{c}\text { Ninth } \\
\text { day } \\
\text { Session } \\
9\end{array}$ & $\begin{array}{l}\text { Principles of proper nutrition } \\
\text { (benefits of proper nutrition, food } \\
\text { groups, factors affecting food } \\
\text { group's requirements, well } \\
\text { balanced diet). }\end{array}$ & $\begin{array}{ll}\text { - } & \text { Discussion } \\
\text { - } & \text { Lecture } \\
\text { - } & \text { Brain storming } \\
\text { - } & \text { Role play } \\
\text { - } & \text { Demonstration } \\
& \text { and } \\
& \text { re-demonstration }\end{array}$ & $\begin{array}{ll}\text { - } & \text { Data show } \\
\text { - } & \text { Real objects } \\
\text { - } & \text { Brochure }\end{array}$ & $\begin{array}{c}1 \\
\text { hour }\end{array}$ & $\begin{array}{ll}> & \text { Pretest } \\
> & \text { Post } \\
& \text { test }\end{array}$ \\
\hline $\begin{array}{c}\text { Tenth } \\
\text { day } \\
\text { Session } \\
10\end{array}$ & $\begin{array}{l}\text { Health maintenance importance } \\
\text { ( definition, factors affecting } \\
\text { health , ways of health } \\
\text { maintenance, benefits of health } \\
\text { maintenance) }\end{array}$ & $\begin{array}{ll}\text { - } & \text { Discussion } \\
\text { - } & \text { Lecture } \\
\text { - } & \text { Brain storming } \\
\text { - } & \text { Role play } \\
\text { - } & \text { Demonstration } \\
& \text { and } \\
& \text { re-demonstration } \\
\end{array}$ & $\begin{array}{ll}\text { - } & \text { Data show } \\
\text { - } & \text { Brochure }\end{array}$ & 1hour & $\begin{aligned} &> \text { Pretest } \\
&> \text { Post } \\
& \text { test }\end{aligned}$ \\
\hline
\end{tabular}




\section{Third Phase: Evaluation of the Educational Intervention}

- Evaluation of the intervention was done two times: immediately and after 3 months of the completion of the intervention for the study group and the control one through posttest structured questionnaire using tool II.

\section{Statistical analysis}

Data were coded and transferred into specially designed formats to be suitable for computer feeding. Following data entry, checking and verifying process were carried out to avoid any errors during data entry. Frequency analysis, cross tabulation and manual revision were all used to detect any errors.

$>$ Data was analyzed using PC with statistical package for social science (SPSS) version 20.

$>$ The level of significance selected for this study was $p$ equal or less than 0.05 .

The next statistical procedures were employed:

1. Descriptive: count, percentage, arithmetic mean, standard deviation.

2. Inferential: the mean change in the overall score of the studied variables was employed for comparison at baseline and follow-up after the educational intervention based on the total maxing allowable score for each variables using: Chi-square test,Fisher's Exact or Monte Carlo correction, Student t-test, ANOVA with repeated measures, Pearson coefficient.

\section{Ethical Considerations:}

- Permission was obtained from ethical committee in the Faculty of Nursing Damnhour University

- Permission was obtained to collect the data from the selected settings.

- Written consent was obtained from every student participated in the study after explanation of the aim of the study and participants were assured that collected data will be used only for the study purpose.

\section{Results}

Table (I) displays distribution of the studied sample according to their personal and socio demographic characteristics. The total number included in the study was 280 (140 cases and 140 control. concerning age, it ranged from 15-18 years with a mean age of $16.39 \pm 0.71$ among the study group whereas among the control group $16.21 \pm 0.71$. Regarding the place of residence, less than two thirds $(63.6 \%)$ of the study group were living in urban areas compared to two thirds $(66.4 \%)$ of the control group. Also, it was evident that the vast majority of both groups $(90 \%)$ had not enough income to meet the demands of their lives.

Table (1):Distribution of The Studied Sample According to Their Socio Demographic Characteristics

\begin{tabular}{|c|c|c|c|c|c|}
\hline \multirow[b]{2}{*}{ Socio-demographic characteristics } & \multicolumn{2}{|c|}{$\begin{array}{l}\text { Study group } \\
(\mathrm{n}=140)\end{array}$} & \multicolumn{2}{|c|}{$\begin{array}{l}\text { Control group } \\
(n=140)\end{array}$} & \multirow{2}{*}{$\begin{array}{c}\text { Test of } \\
\text { Significance }\end{array}$} \\
\hline & No. & $\%$ & No. & $\%$ & \\
\hline \multicolumn{6}{|l|}{ Age in years } \\
\hline $15<16$ & 13 & 9.3 & 24 & 17.1 & \\
\hline $16<17$ & 65 & 46.4 & 67 & 47.9 & $\chi^{2}=4.974$ \\
\hline $17<18$ & 57 & 40.7 & 44 & 31.4 & ${ }^{\mathrm{MC}} \mathrm{p}=0.174$ \\
\hline $18<19$ & 5 & 3.6 & 5 & 3.6 & \\
\hline $\begin{array}{l}\text { Min. - Max. } \\
\text { Mean } \pm \text { SD. }\end{array}$ & \multicolumn{2}{|c|}{$\begin{array}{c}15.0-18.0 \\
16.39 \pm 0.71\end{array}$} & \multicolumn{2}{|c|}{$\begin{array}{c}15.0-18.0 \\
16.21 \pm 0.71\end{array}$} & $\begin{array}{c}\mathrm{t}=1.948 \\
\mathrm{MC}_{\mathrm{p}}=0.052\end{array}$ \\
\hline \multicolumn{6}{|l|}{ Residence } \\
\hline Urban & 89 & 63.6 & 93 & 66.4 & $\chi^{2}=0.251$ \\
\hline Rural & 51 & 36.4 & 47 & 33.6 & ${ }^{M}{ }_{p}=0.616$ \\
\hline \multicolumn{6}{|l|}{ Family income } \\
\hline Enough & 14 & 10.0 & 14 & 10.0 & $\chi^{2}=0.000$ \\
\hline Not enough & 126 & 90.0 & 126 & 90.0 & ${ }^{\mathrm{MC}} \mathrm{p}=1.000$ \\
\hline \multicolumn{6}{|l|}{ Crowding index } \\
\hline$<2$ & 10 & 7.1 & 5 & 3.6 & \\
\hline $2-<4$ & 30 & 21.4 & 40 & 28.6 & $\begin{array}{c}\chi^{2}=11.497 \\
\mathrm{MC}_{n}=0001^{*}\end{array}$ \\
\hline$\geq 4$ & 100 & 71.5 & 95 & 67.8 & $\mathrm{p}=0.001$ \\
\hline $\begin{array}{l}\text { Min. - Max. } \\
\text { Mean } \pm \text { SD. }\end{array}$ & \multicolumn{2}{|c|}{$\begin{array}{c}1-10 \\
2.4+0.9\end{array}$} & \multicolumn{2}{|c|}{$\begin{array}{c}1-5 \\
2.1 \pm 0.6\end{array}$} & $\begin{array}{l}\mathrm{t}=3.627^{*} \\
\mathrm{p}<0.001^{*}\end{array}$ \\
\hline
\end{tabular}




\begin{tabular}{|c|c|c|c|c|c|}
\hline \multirow[b]{2}{*}{ Socio-demographic characteristics } & \multicolumn{2}{|c|}{$\begin{array}{l}\text { Study group } \\
(\mathrm{n}=140)\end{array}$} & \multicolumn{2}{|c|}{$\begin{array}{c}\text { Control group } \\
(n=140)\end{array}$} & \multirow{2}{*}{$\begin{array}{c}\text { Test of } \\
\text { Significance }\end{array}$} \\
\hline & No. & $\%$ & No. & $\%$ & \\
\hline \multicolumn{6}{|l|}{ Mother Level of education } \\
\hline Illiterate & 13 & 9.3 & 14 & 10.0 & \multirow{6}{*}{$\begin{array}{c}\chi^{2}=4.225 \\
{ }^{M C} \mathrm{p}=0.524\end{array}$} \\
\hline Read and write & 3 & 2.1 & 3 & 2.1 & \\
\hline Primary school & 5 & 3.6 & 3 & 2.1 & \\
\hline Preparatory school & 10 & 7.1 & 12 & 8.6 & \\
\hline Secondary school & 69 & 49.3 & 55 & 39.3 & \\
\hline University education & 40 & 28.6 & 53 & 37.9 & \\
\hline
\end{tabular}

$\chi^{2}$ : Chi square test MC: Monte Carlo

p: $\mathrm{p}$ value for comparing between Experimental and Control

*: Statistically significant at $\mathrm{p} \leq 0.05$

Table ( I): (CONT.) :

\begin{tabular}{|c|c|c|c|c|c|}
\hline \multirow[t]{2}{*}{ Socio-demographic characteristics } & \multicolumn{2}{|c|}{$\begin{array}{l}\text { Study group } \\
\quad(\mathrm{n}=140)\end{array}$} & \multicolumn{2}{|c|}{$\begin{array}{ll}\begin{array}{l}\text { Control } \\
(n=140)\end{array} & \text { group } \\
\end{array}$} & \multirow{2}{*}{$\begin{array}{c}\text { Test of } \\
\text { Significance }\end{array}$} \\
\hline & No. & $\%$ & No. & $\%$ & \\
\hline \multicolumn{6}{|l|}{ Father level of education } \\
\hline Illiterate & 5 & 3.6 & 5 & 3.6 & \multirow{6}{*}{$\begin{aligned} \chi^{2} & =5.839 \\
{ }^{\mathrm{MC}} \mathrm{p} & =0.304\end{aligned}$} \\
\hline Read and write & 1 & 0.7 & 0 & 0.0 & \\
\hline Primary school & 1 & 0.7 & 4 & 2.9 & \\
\hline Preparatory school & 15 & 10.7 & 12 & 8.5 & \\
\hline Secondary school & 46 & 32.9 & 34 & 24.3 & \\
\hline University education & 72 & 51.4 & 85 & 60.7 & \\
\hline \multicolumn{6}{|l|}{ Mother's occupation } \\
\hline House wife & 74 & 52.9 & 57 & 40.7 & \multirow{6}{*}{$\begin{array}{l}\chi^{2}=23.635^{*} \\
{ }_{\mathrm{MC}}<<0.001^{*}\end{array}$} \\
\hline Unskilled manual worker & 3 & 2.1 & 0 & 0.0 & \\
\hline Farmer & 5 & 3.5 & 0 & 0.0 & \\
\hline Trades/business & 27 & 19.3 & 23 & 16.5 & \\
\hline Semiprofessional /clerk & 6 & 4.3 & 3 & 2.1 & \\
\hline Professional & 25 & 17.9 & 57 & 40.7 & \\
\hline \multicolumn{6}{|l|}{ Father's occupation } \\
\hline Non-working/retired & 2 & 1.5 & 13 & 9.3 & \multirow{6}{*}{$\begin{array}{l}\chi^{2}=17.581^{*} \\
{ }_{\mathrm{MC}} \mathrm{p}<0.001^{*}\end{array}$} \\
\hline Unskilled manual worker & 5 & 3.6 & 4 & 2.9 & \\
\hline Skilled manual worker/farmer & 5 & 3.6 & 0 & 0.0 & \\
\hline Trades/business & 45 & 32.1 & 29 & 20.7 & \\
\hline Semiprofessional / clerk & 3 & 2.1 & 4 & 2.9 & \\
\hline Professional & 80 & 57.1 & 90 & 64.2 & \\
\hline
\end{tabular}

$\chi^{2}$ : Chi square test MC: Monte Carlo

p: $p$ value for comparing between Experimental and Control

*: Statistically significant at $\mathrm{p} \leq 0.05$

Table (II) Illustrates distribution of the studied sample according to their life style pattern. Concerning the dietary pattern, around two thirds of the study group (61.4\%) reported that they had three meals per day compared to more than two thirds $(69.3 \%)$ of the control group. The table also portrays that nearly two fifths $(41.4 \%)$ of the study group always had breakfast daily compared to more than half $(52.9 \%)$ of the control group. Additionally, it was found that half of the study group ( 50\%) reported always intake of fast food compared to less than two third $(63.6 \%)$ of the control group. Moreover, it was apparent that the vast majority of the study group were consuming beverages.

Concerning exercise, it was evident that, less than two thirds (60.7\%) of the study group didn't practice any type of exercise compared to more than three quarters $(83.6 \%)$ of the control group, whereas the rest of both groups were practicing exercises.

With respect to risk-taking behaviors, the table depicts that more than one third (37.1) of the study group reported that they were smokers compared to about one fifth $(20.7 \%)$ of the control group. 
Table (II):Distribution of the Studied Sample According to their life style pattern

\begin{tabular}{|c|c|c|c|c|c|}
\hline \multirow[t]{2}{*}{ Life style pattern } & \multicolumn{2}{|c|}{$\begin{array}{c}\text { Study group } \\
(\mathrm{n}=140)\end{array}$} & \multicolumn{2}{|c|}{$\begin{array}{c}\text { Control group } \\
(\mathrm{n}=140)\end{array}$} & \multirow{2}{*}{$\begin{array}{c}\text { Test of } \\
\text { Significance }\end{array}$} \\
\hline & No. & $\%$ & No. & $\%$ & \\
\hline \multicolumn{6}{|l|}{ A. Eating habits } \\
\hline \multicolumn{6}{|l|}{ Number of meals per day } \\
\hline One meal & 0 & 0.0 & 0 & 0.0 & \multirow{4}{*}{$\begin{array}{c}\chi^{2}=4.893 \\
{ }_{\mathrm{MC}}=0.070\end{array}$} \\
\hline Two meals & 4 & 2.9 & 0 & 0.0 & \\
\hline Three meals & 86 & 61.4 & 97 & 69.3 & \\
\hline More than three & 50 & 35.7 & 43 & 30.7 & \\
\hline \multicolumn{6}{|l|}{ Intake of breakfast meal } \\
\hline Always & 58 & 41.4 & 74 & 52.9 & \multirow{3}{*}{$\begin{array}{c}\chi^{2}=13.576^{*} \\
{ }_{\mathrm{MC}} \mathrm{p}=0.001^{*}\end{array}$} \\
\hline Sometimes & 63 & 45.0 & 63 & 45.0 & \\
\hline Never & 19 & 13.6 & 3 & 2.1 & \\
\hline \multicolumn{6}{|l|}{ Fast food intake } \\
\hline Always & 70 & 50.0 & 89 & 63.6 & \multirow{3}{*}{$\begin{array}{c}\chi^{2}=14.269^{*} \\
{ }_{\mathrm{MC}} \mathrm{p}=0.001^{*}\end{array}$} \\
\hline Sometimes & 53 & 37.9 & 49 & 35.0 & \\
\hline Never & 17 & 12.1 & 2 & 1.4 & \\
\hline \multicolumn{6}{|l|}{ Number of water glasses per day } \\
\hline$<8$ cups/ day & 58 & 41.4 & 80 & 57.1 & \multirow{3}{*}{$\begin{array}{c}\chi^{2}=12.267^{*} \\
{ }^{\mathrm{MC}} \mathrm{p}=0.002^{*}\end{array}$} \\
\hline 8 cups/ day & 61 & 43.6 & 54 & 38.6 & \\
\hline$>8$ cups/ day & 21 & 15.0 & 6 & 4.3 & \\
\hline Mean \pm SD & \multicolumn{2}{|c|}{$9.86 \pm 3.07$} & \multicolumn{2}{|c|}{$10.56 \pm 3.07$} & $\begin{array}{c}\mathrm{t}=3.627^{*} \\
\mathrm{MC}_{\mathrm{p}}<0.001^{*}\end{array}$ \\
\hline \multicolumn{6}{|l|}{ Beverages intake } \\
\hline No & 14 & 10.0 & 0 & 0.0 & $\chi^{2}=14.737^{*}$ \\
\hline Yes & 126 & 90.0 & 140 & 100.0 & ${ }^{\mathrm{M}} \mathrm{p}<0.001$ \\
\hline Beverages intake frequency & \multicolumn{2}{|c|}{$n=(126)$} & \multicolumn{2}{|c|}{$n=(140)$} & \\
\hline Three times per day & 54 & 42.9 & 65 & 46.4 & \\
\hline Two times per day & 55 & 43.6 & 64 & 45.7 & $\chi^{2}=12.267$ \\
\hline More than three times per day & 17 & 13.5 & 11 & 7.9 & $p=0.002$ \\
\hline
\end{tabular}

$\chi^{2}$ : Chi square test

MC: Monte Carlo

$\mathrm{p}: \mathrm{p}$ value for comparing between the studied groups

*: Statistically significant at $\mathrm{p} \leq 0.05$

Table (II): (CONT.) :

\begin{tabular}{|c|c|c|c|c|c|}
\hline \multirow[t]{2}{*}{ Life style pattern } & \multicolumn{2}{|c|}{$\begin{array}{ll}\text { Study } & \text { group } \\
(\mathrm{n}=140) & \end{array}$} & \multicolumn{2}{|c|}{$\begin{array}{|ll|}\begin{array}{l}\text { Control } \\
(n=140)\end{array} & \text { group } \\
\end{array}$} & \multirow{2}{*}{$\begin{array}{l}\text { Test } \\
\text { Significance }\end{array}$} \\
\hline & No. & $\%$ & No. & $\%$ & \\
\hline \multicolumn{6}{|l|}{ B. Exercise } \\
\hline \multicolumn{6}{|l|}{ Practicing exercise } \\
\hline $\begin{array}{l}\text { No } \\
\text { Yes }\end{array}$ & $\begin{array}{l}85 \\
55\end{array}$ & $\begin{array}{l}60.7 \\
39.3\end{array}$ & $\begin{array}{l}117 \\
23\end{array}$ & \begin{tabular}{|l|}
83.6 \\
16.4
\end{tabular} & $\begin{array}{l}\chi^{2}=18.198^{*} \\
\mathrm{MC}_{\mathrm{p}}<0.001^{*}\end{array}$ \\
\hline $\begin{array}{l}\text { Frequency of practicing exercise per } \\
\text { week }\end{array}$ & \multicolumn{2}{|c|}{$(n=55)$} & \multicolumn{2}{|c|}{$(n=23)$} & \\
\hline $\begin{array}{l}\text { Once } \\
\text { Twice } \\
\text { More than twice }\end{array}$ & $\begin{array}{l}51 \\
4 \\
0 \\
\end{array}$ & $\begin{array}{l}92.7 \\
7.3 \\
0.0 \\
\end{array}$ & $\begin{array}{l}15 \\
8 \\
0 \\
\end{array}$ & \begin{tabular}{|l|}
65.2 \\
34.8 \\
0.0 \\
\end{tabular} & $\begin{array}{l}\chi^{2}=26.039^{*} \\
{ }^{\mathrm{MC}} \mathrm{p}<0.001^{*}\end{array}$ \\
\hline \multicolumn{6}{|l|}{ Causes of not practicing exercise } \\
\hline $\begin{array}{l}\text { Lack of time } \\
\text { Sickness reasons }\end{array}$ & $\begin{array}{l}50 \\
5\end{array}$ & $\begin{array}{l}90.9 \\
9.1 \\
\end{array}$ & $\begin{array}{l}19 \\
4\end{array}$ & $\begin{array}{l}82.6 \\
17.4\end{array}$ & $\begin{array}{l}\chi^{2}=16.014^{*} \\
{ }_{\mathrm{MC}} \mathrm{p}<0.001^{*}\end{array}$ \\
\hline
\end{tabular}




\begin{tabular}{|c|c|c|c|c|c|}
\hline \multirow[t]{2}{*}{ Life style pattern } & \multicolumn{2}{|c|}{\begin{tabular}{|ll} 
Study & group \\
$(\mathrm{n}=140)$ &
\end{tabular}} & \multicolumn{2}{|c|}{$\begin{array}{ll}\begin{array}{l}\text { Control } \\
(\mathrm{n}=140)\end{array} & \text { group } \\
\end{array}$} & \multirow{2}{*}{$\begin{array}{l}\text { Test } \\
\text { Significance }\end{array}$} \\
\hline & No. & $\%$ & No. & $\%$ & \\
\hline \multicolumn{6}{|l|}{ C. Sleeping pattern } \\
\hline \multicolumn{6}{|c|}{ Sleeping at night ( per hours) } \\
\hline$<6$ hours & 85 & 60.7 & 80 & 57.1 & $\chi^{2}=0.369$ \\
\hline$\geq 6$ hours & 55 & 39.3 & 60 & 42.9 & ${ }^{\mathrm{MC}} \mathrm{p}=0.544$ \\
\hline Min. - Max. & \multirow{2}{*}{\multicolumn{2}{|c|}{$\begin{array}{l}4.0-9.0 \\
5.75 \pm 1.68\end{array}$}} & \multirow{2}{*}{\multicolumn{2}{|c|}{$\begin{array}{l}4.0-8.0 \\
5.59 \pm 1.40\end{array}$}} & $\mathrm{t}=0.851$ \\
\hline Mean $\pm \mathrm{SD}$ & & & & & ${ }^{\mathrm{MC}} \mathrm{p}=0.396$ \\
\hline \multicolumn{6}{|c|}{ Presence of sleep problems } \\
\hline No & 125 & 89.3 & 72 & 51.4 & $\chi^{2}=48.102^{*}$ \\
\hline Yes & 15 & 10.7 & 68 & 48.6 & ${ }^{\mathrm{MC}} \mathrm{p}<0.001^{*}$ \\
\hline Type of sleep problems & \multicolumn{2}{|c|}{$(n=15)$} & \multicolumn{2}{|c|}{$(n=68)$} & \\
\hline Sleeping a lot & 7 & 46.7 & 12 & 17.6 & \\
\hline Difficulty in sleeping & 6 & 40 & 39 & 57.4 & $\chi^{2}=51.617$ \\
\hline Disturbed sleep & 2 & 13.3 & 17 & 25 & \\
\hline Others & 0 & 0.0 & 0 & 0.0 & \\
\hline
\end{tabular}

$\chi^{2}$ : Chi square test

t: Student t-test

$\mathrm{p}$ : $\mathrm{p}$ value for comparing between the studied groups

$*$ : Statistically significant at $\mathrm{p} \leq 0.05$

Table (II): (CONT.) :

\begin{tabular}{|c|c|c|c|c|c|}
\hline \multirow[t]{2}{*}{ Life style pattern } & \multicolumn{2}{|c|}{$\begin{array}{l}\text { Study group } \\
(\mathrm{n}=140)\end{array}$} & \multicolumn{2}{|c|}{$\begin{array}{c}\text { Control group } \\
(n=140)\end{array}$} & \multirow{2}{*}{$\begin{array}{c}\text { Test of } \\
\text { significance }\end{array}$} \\
\hline & No. & $\%$ & No. & $\%$ & \\
\hline \multicolumn{6}{|l|}{ D. Risk taking behaviors } \\
\hline \multicolumn{6}{|l|}{ Students' smoking habit } \\
\hline No & 88 & 62.9 & 111 & 79.3 & $\chi^{2}=9.189^{*}$ \\
\hline Yes & 52 & 37.1 & 29 & 20.7 & ${ }^{\mathrm{MC}} \mathrm{p}=0.002^{*}$ \\
\hline Duration of starting smoking & \multicolumn{2}{|c|}{$(n=52)$} & \multicolumn{2}{|c|}{$(n=29)$} & \\
\hline 2 years ago & 22 & 42.3 & 17 & 58.6 & $\chi^{2}=17.547^{*}$ \\
\hline 1 year ago & 20 & 38.5 & 12 & 41.4 & ${ }^{\mathrm{MC}} \mathrm{p}=0.001^{*}$ \\
\hline Three years ago & 10 & 19.2 & 0 & 0.0 & \\
\hline \multicolumn{6}{|l|}{ Number of cigarettes per day } \\
\hline $5-$ & 34 & 65.4 & 21 & 72.4 & \multirow{3}{*}{$\begin{array}{c}\chi^{2}=13.403^{*} \\
{ }^{\mathrm{MC}} \mathrm{p}=0.003^{*}\end{array}$} \\
\hline $7-$ & 14 & 26.9 & 8 & 27.6 & \\
\hline $9+$ & 4 & 7.7 & 0 & 0.0 & \\
\hline Min. - Max. & \multicolumn{2}{|c|}{$5.0-9.0$} & \multicolumn{2}{|c|}{$5.0-7.0$} & \multirow{2}{*}{$\begin{array}{l}\mathrm{t}=1.488 \\
\mathrm{p}=0.141\end{array}$} \\
\hline Mean \pm SD. & \multicolumn{2}{|c|}{$5.91 \pm 1.27$} & \multicolumn{2}{|c|}{$5.55 \pm 0.91$} & \\
\hline \multicolumn{6}{|l|}{ Taking drugs } \\
\hline No & 140 & 100.0 & 140 & 100.0 & \multirow[b]{2}{*}{-} \\
\hline Yes & 0 & 0.0 & 0 & 0.0 & \\
\hline
\end{tabular}

$\chi^{2}$ : Chi square test

t: Student t-test

$\mathrm{p}$ : $\mathrm{p}$ value for comparing between the studied groups

*: Statistically significant at $\mathrm{p} \leq 0.05$

Table (III): Reveals Distribution of the Studied Sample According To Their Total Knowledge Score before and After Program. It is apparent from the table that concerning occupational health and safety in the school before the educational intervention all the study group and the control one scored poorly. Conversely, after the educational intervention, more than half (52.8\%) of the study group had good knowledge and more than one tenth $(13.6 \%)$ had fair knowledge, whilst one third of them still had poor knowledge. However, the entire control group still had poor knowledge, with a statistically significant difference between both groups $\left(\mathrm{X}^{2}=\right.$ 139.251, $\left.{ }^{\mathrm{MC}} \mathrm{p}=<0.001\right)$.

Pertaining to occupational hazards, the table shows that before the educational intervention, the vast 
majority of both groups $(97.1 \%, 98.6 \%)$ had poor knowledge, and the rest of both groups $(2.9 \%, 1.4 \%)$ had fair knowledge, with a statistically significant difference between both groups $\left(\mathrm{X}^{2}=6.272,{ }^{\mathrm{FE}} \mathrm{p}=0.684\right)$. By contrast, after the educational intervention, more than one third $(41.4 \%)$ of the study group had good knowledge compared to only $0.7 \%$ of the control one. Additionally, less than half $(47.9 \%)$ of the study group had fair knowledge compared to just $2.1 \%$ of the control group. Moreover, around one tenth $(10.7 \%)$ of the study group had poor knowledge, whereas the vast majority of the control group still had poor knowledge, with astatically significant difference between both groups $\left(\mathrm{X}^{2}=210.542,{ }^{\mathrm{MC}} \mathrm{p}=<0.001\right)$.

In relation to school accidents, the table conveys that before the educational intervention the vast majority $(92.9 \%, 97.1 \%)$ of the study group and the control one respectively scored poorly and the minority of both groups $(2.9 \%)$ had good knowledge, with a statistically significant difference between both groups $\left(\mathrm{X}^{2}=\right.$ $\left.6.272,{ }^{\mathrm{MC}} \mathrm{p}=0.047\right)$. Otherwise, after the educational intervention, two-fifth $(40 \%)$ of the study group had correct knowledge compared to a minority $(2.9 \%)$ of the control group .In addition, more than one third $(37.9 \%)$ of the study group had fair knowledge compared to only $2.9 \%$ of the control one. As well as that, less than one quarter $(22.1 \%)$ of the study group had poor knowledge, whilst the vast majority(94.2\%) of the control group still had poor knowledge, with astatically significant difference between both groups $\left(\mathrm{X}^{2}=149.77,{ }^{\mathrm{MC}} \mathrm{p}=<0.001\right)$.

The table also represented that before the educational intervention all the study group and the control one had poor knowledge regarding safety measures that must be available in the workshop. Nevertheless, after the educational intervention, more than two thirds $(68.5 \%)$ of the study group had good knowledge, whilst more than one fifth $(23.6 \%)$ of them had fair knowledge and the rest of them $(7.9 \%)$ had poor knowledge. Furthermore, all the control group still had poor knowledge, with a statistically significant difference between both groups $\left(\mathrm{X}^{2}=\right.$ 239.205, $\left.{ }^{\mathrm{MC}} \mathrm{p}=<0.001\right)$.

The table also depicts that all the study group and the vast majority (98.6\%) of the control one had poor knowledge regarding first aids before the educational intervention, with no statistically significant difference between both groups $\left(\mathrm{X}^{2}=2.014,{ }^{\mathrm{FE}} \mathrm{p}=0.498\right)$. Unlike, after the educational intervention, less than two thirds $(63.6 \%)$ of the study group had good knowledge, whereas less than two fifth $(15.7 \%)$ had fair knowledge and the rest of them $(20.7 \%)$ had poor knowledge. Along with, the vast majority $(98.6 \%)$ of the control group and only $1.4 \%$ of them had fair knowledge. A statistically significant difference was found between both groups $\left(X^{2}=\right.$ $\left.176.320,{ }^{\mathrm{MC}} \mathrm{p}=<0.001\right)$.

Table (III): Distribution of the Studied Sample According To Their Total Knowledge Score before and After Program

\begin{tabular}{|c|c|c|c|c|c|c|c|c|}
\hline \multirow{3}{*}{ Knowledge Level } & \multicolumn{4}{|c|}{ Before } & \multicolumn{4}{|c|}{ After } \\
\hline & \multicolumn{2}{|c|}{$\begin{array}{c}\text { Study } \\
\text { group } \\
(\mathrm{n}=\mathbf{1 4 0})\end{array}$} & \multicolumn{2}{|c|}{$\begin{array}{l}\text { Control group } \\
\quad(n=140)\end{array}$} & \multicolumn{2}{|c|}{$\begin{array}{l}\text { Study group } \\
\quad(\mathrm{n}=140)\end{array}$} & \multicolumn{2}{|c|}{$\begin{array}{c}\text { Control } \\
\text { group } \\
(n=140)\end{array}$} \\
\hline & No. & $\%$ & No. & $\%$ & No. & $\%$ & No. & $\%$ \\
\hline \multicolumn{9}{|c|}{ Occupational health and safety in the school } \\
\hline Poor & 140 & 100.0 & 140 & 100.0 & 47 & 33.6 & 140 & 100.0 \\
\hline Fair & 0 & 0.0 & 0 & 0.0 & 19 & 13.6 & 0 & 0.0 \\
\hline Good & 0 & 0.0 & 0 & 0.0 & 74 & 52.8 & 0 & 0.0 \\
\hline$\chi^{2}(\mathrm{p})$ & \multicolumn{4}{|c|}{-} & \multicolumn{2}{|c|}{$\begin{array}{l}139.251^{*} \\
\left(<0.001^{*}\right)\end{array}$} & & \\
\hline \multicolumn{9}{|l|}{ Occupational hazards } \\
\hline Poor & 136 & 97.1 & 138 & 98.6 & 15 & 10.7 & 136 & 97.2 \\
\hline Fair & 4 & 2.9 & 2 & 1.4 & 67 & 47.9 & 3 & 2.1 \\
\hline Good & 0 & 0.0 & 0 & 0.0 & 58 & 41.4 & 1 & 0.7 \\
\hline$\chi^{2}(\mathrm{p})$ & \multicolumn{4}{|c|}{$0.681\left({ }^{F E} p=0.684\right)$} & \multicolumn{4}{|c|}{$210.542^{*}\left(<0.001^{*}\right)$} \\
\hline \multicolumn{9}{|l|}{ School accidents } \\
\hline Poor & 130 & 92.9 & 136 & 97.1 & 31 & 22.1 & 132 & 94.2 \\
\hline Fair & 6 & 4.2 & 0 & 0.0 & 53 & 37.9 & 4 & 2.9 \\
\hline Good & 4 & 2.9 & 4 & 2.9 & 56 & 40.0 & 4 & 2.9 \\
\hline$\chi^{2}(\mathrm{p})$ & \multicolumn{4}{|c|}{$6.272\left({ }^{\mathrm{MC}} \mathrm{p}=0.047^{*}\right)$} & \multicolumn{4}{|c|}{$149.772^{*}\left(<0.001^{*}\right)$} \\
\hline \multicolumn{9}{|c|}{$\begin{array}{l}\text { Safety measures that must be available in the } \\
\text { workshop }\end{array}$} \\
\hline Poor & 140 & 100.0 & 140 & 100.0 & 11 & 7.9 & 140 & 100.0 \\
\hline Fair & 0 & 0.0 & 0 & 0.0 & 33 & 23.6 & 0 & 0.0 \\
\hline Good & 0 & 0.0 & 0 & 0.0 & 96 & 68.5 & 0 & 0.0 \\
\hline$\chi^{2}(p)$ & \multicolumn{4}{|c|}{-} & \multicolumn{4}{|c|}{$239.205^{*}\left(<0.001^{*}\right)$} \\
\hline
\end{tabular}




\begin{tabular}{|c|c|c|c|c|c|c|c|c|}
\hline \multirow{3}{*}{ Knowledge Level } & \multicolumn{4}{|c|}{ Before } & \multicolumn{4}{|c|}{ After } \\
\hline & \multicolumn{2}{|c|}{$\begin{array}{c}\text { Study } \\
\text { group } \\
(\mathrm{n}=140)\end{array}$} & \multicolumn{2}{|c|}{$\begin{array}{l}\text { Control group } \\
\quad(n=140)\end{array}$} & \multicolumn{2}{|c|}{$\begin{array}{l}\text { Study group } \\
\quad(\mathrm{n}=140)\end{array}$} & \multicolumn{2}{|c|}{$\begin{array}{c}\text { Control } \\
\text { group } \\
(\mathrm{n}=140)\end{array}$} \\
\hline & No. & $\%$ & No. & $\%$ & No. & $\%$ & No. & $\%$ \\
\hline \multicolumn{9}{|l|}{ First aids } \\
\hline Poor & 140 & 100.0 & 138 & 98.6 & 29 & 20.7 & 138 & 98.6 \\
\hline Fair & 0 & 0.0 & 2 & 1.4 & 22 & 15.7 & 2 & 1.4 \\
\hline Good & 0 & 0.0 & 0 & 0.0 & 89 & 63.6 & 0 & 0.0 \\
\hline$\chi^{2}(\mathrm{p})$ & \multicolumn{4}{|c|}{$2.014\left({ }^{\mathrm{FE}} p=0.498\right)$} & \multicolumn{2}{|c|}{$\begin{array}{l}176.320^{*} \\
\left(<0.001^{*}\right)\end{array}$} & & \\
\hline
\end{tabular}

$\chi^{2}$ : Chi square test MC: Monte Carlo

value for comparing between Experimental and Control

*: Statistically significant at $\mathrm{p} \leq 0.05$

FE: Fisher Exact p: $p$

Figure ( I): Study group overall knowledge scores before and after program implementation.

It is clear from the figure that before conduction of the educational intervention, all the study group had poor knowledge, while after the program implementation, the majority of the students had fair knowledge.

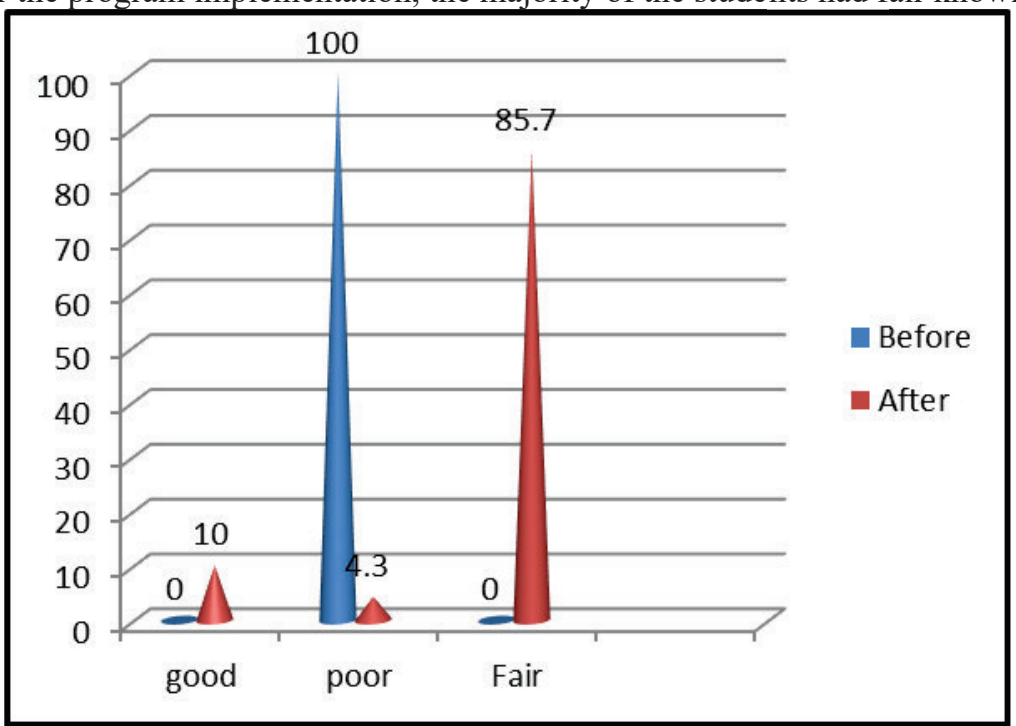

Figure (II): Control group overall knowledge scores before and after program implementation. The figure conveys that the control group had poor knowledge before and after implementation of the educational intervention.

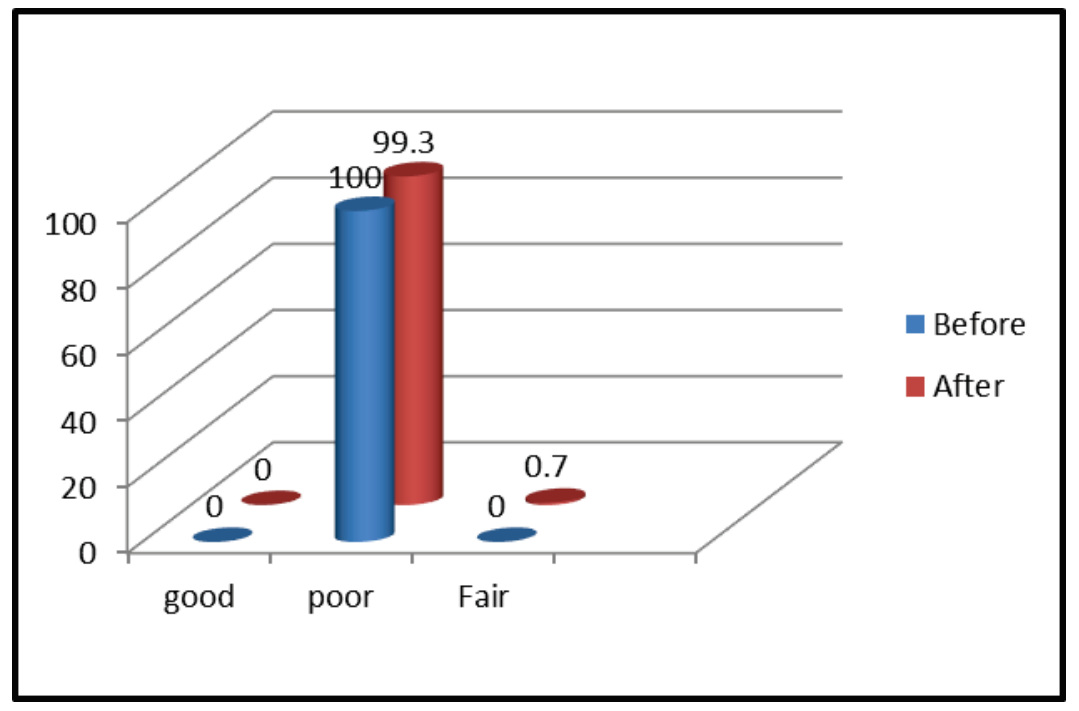


Figure (III) Portrays Distribution Of The Studied Sample According To Their Overall Total Mean Score. It is apparent from this figure that the overall mean score for the study group before the educational intervention was $(12.79 \pm 7.99)$, whereas the control group was $(13.03 \pm 8.26)$, with no statistically significant difference between both groups ( $\mathrm{t}$-test $=0.243, \mathrm{P}=0.808)$. On the contrary, after the educational intervention, the mean score for the study group rose to $(79.24 \pm 11.62)$, whereas the control group remained the same $(13.20 \pm 8.27)$, with a statistically significant difference between both groups $(\mathrm{t}$-test $=54.792, \mathrm{P}=<0.001)$. Additionally, three months after the educational intervention, the mean score for the study group was $(78.79 \pm 11.49)$, whereas the control group was $(14.10 \pm 9.18)$, with a statically significant difference between both groups ( $\mathrm{t}$-test $=52.037, \mathrm{P}$ $=<0.001$ ).

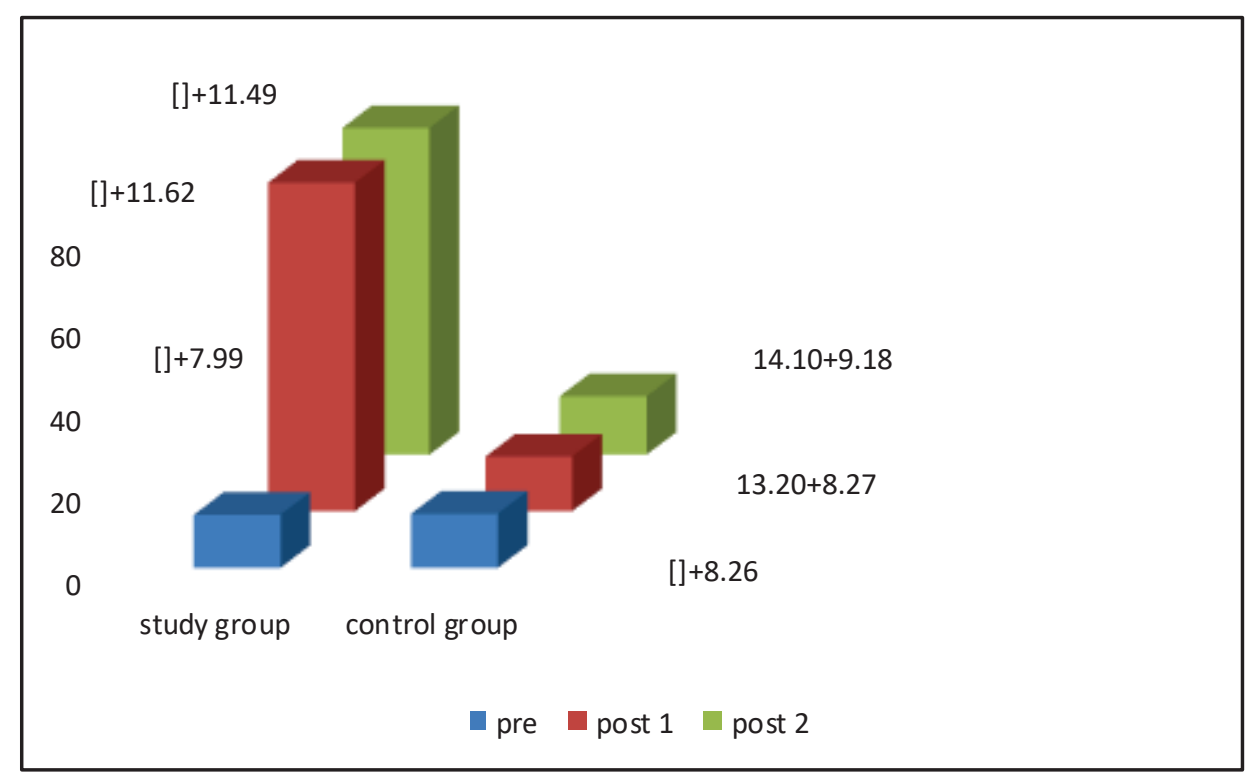

Table (IV): Puzzles out the Relation between the students' knowledge level and their socio-demographic characteristics. It was apparent from the table that there was significant correlation between age, department and level of knowledge. $\left(\mathrm{X}^{2}=15.929, \mathrm{P}=0.014^{*}\right),\left(\mathrm{X}^{2}=14.150, \mathrm{P}=0.007^{*}\right)$. Nevertheless , It can also observed from the table that factors such as place of residence, family income, crowding index, Mother's level of education, Mother's occupation, Father's level of education, and Father's occupation of the studied sample had an insignificant effect on their knowledge score. $\left(\mathrm{X}^{2}=1.897, \mathrm{P}=0.387, \mathrm{X}^{2}=2.593, \mathrm{P}=0.274, \mathrm{X}^{2}=0.809\right.$, $\left.\mathrm{P}=0.667, \mathrm{X}^{2}=9.028, \mathrm{P}=0.529, \mathrm{X}^{2}=5.503, \mathrm{P}=0.064, \mathrm{X}^{2}=4.377, \mathrm{P}=0.929, \mathrm{X}^{2}=0.338, \mathrm{P}=0.844\right)$.

Table (V): Relation between the students' knowledge level and their socio demographic characteristics

\begin{tabular}{|c|c|c|c|c|c|c|c|c|c|}
\hline \multirow[t]{3}{*}{ Item } & \multicolumn{6}{|c|}{ Knowledge level } & \multirow{2}{*}{\multicolumn{2}{|c|}{$\begin{array}{r}\text { Total } \\
n=140\end{array}$}} & \multirow{3}{*}{$\begin{array}{c}\text { Test of } \\
\text { significance }\end{array}$} \\
\hline & \multicolumn{2}{|c|}{$\begin{array}{l}\text { Poor } \\
(n=6)\end{array}$} & \multicolumn{2}{|c|}{$\begin{array}{c}\text { Fair } \\
(n=120)\end{array}$} & \multicolumn{2}{|c|}{$\begin{array}{r}\text { Good } \\
(n=14)\end{array}$} & & & \\
\hline & No. & $\%$ & No. & $\%$ & No. & $\%$ & & & \\
\hline \multicolumn{10}{|l|}{ Age } \\
\hline$-15-$ & 1 & 7.7 & 8 & 61.5 & 4 & 30.8 & 13 & 9.3 & \multirow{4}{*}{$\begin{array}{l}X^{2}=15.929 \\
P=0.014^{*}\end{array}$} \\
\hline$-16-$ & 1 & 1.5 & 59 & 90.8 & 5 & 7.7 & 65 & 46.4 & \\
\hline$-17-$ & 4 & 7.0 & 50 & 87.7 & 3 & 5.3 & 57 & 40.7 & \\
\hline$-18-<19$ & 0 & 0.0 & 3 & 60.0 & 2 & 40.0 & 5 & 3.6 & \\
\hline \multicolumn{10}{|c|}{ Department } \\
\hline - Welding & 1 & 1.3 & 63 & 82.9 & 12 & 15.8 & 76 & 54.3 & \multirow{3}{*}{$\begin{array}{l}X^{2}=14.150 \\
P=0.007^{*}\end{array}$} \\
\hline - Lathing & 5 & 11.9 & 36 & 85.7 & 1 & 2.4 & 42 & 30.0 & \\
\hline - Foundry & 0 & 0.0 & 21 & 95.5 & 1 & 4.5 & 22 & 15.7 & \\
\hline \multicolumn{10}{|c|}{ Place of residence } \\
\hline - Urban & 3 & 3.4 & 75 & 84.3 & 11 & 12.4 & 89 & 63.6 & $X^{2}=1.897$ \\
\hline - Rural & 3 & 5.9 & 45 & 88.2 & 3 & 5.9 & 51 & 36.4 & $\mathrm{P}=0.387$ \\
\hline
\end{tabular}




\begin{tabular}{|c|c|c|c|c|c|c|c|c|c|}
\hline \multirow[t]{3}{*}{ Item } & \multicolumn{6}{|c|}{ Knowledge level } & \multirow{2}{*}{\multicolumn{2}{|c|}{$\begin{array}{r}\text { Total } \\
n=140\end{array}$}} & \multirow[t]{3}{*}{$\begin{array}{c}\text { Test of } \\
\text { significance }\end{array}$} \\
\hline & \multicolumn{2}{|c|}{$\begin{array}{l}\text { Poor } \\
(n=6)\end{array}$} & \multicolumn{2}{|c|}{$\begin{array}{c}\text { Fair } \\
(n=120)\end{array}$} & \multicolumn{2}{|c|}{$\begin{array}{r}\text { Good } \\
(n=14)\end{array}$} & & & \\
\hline & No. & $\%$ & No. & $\%$ & No. & $\%$ & & & \\
\hline \multicolumn{10}{|l|}{ Family income } \\
\hline - Enough & 0 & 0.0 & 14 & 100.0 & 0 & 0.0 & 14 & 10.0 & $X^{2}=2.593$ \\
\hline - Not enough & 6 & 4.8 & 106 & 84.1 & 14 & 11.1 & 126 & 90.0 & $\mathrm{P}=0.274$ \\
\hline \multicolumn{10}{|l|}{ Crowding index } \\
\hline$-<1.5$ & 2 & 3.6 & 46 & 83.6 & 7 & 12.7 & 55 & 39.3 & $x^{2}=0.809$ \\
\hline$-\geq 1.5$ & 4 & 4.7 & 74 & 87.1 & 7 & 8.2 & 85 & 60.7 & $\mathrm{P}=0.667$ \\
\hline \multicolumn{10}{|c|}{ Mother's level of education } \\
\hline - Illiterate & 0 & 0.0 & 11 & 84.6 & 2 & 15.4 & 13 & 9.3 & $x^{2}=9.028$ \\
\hline - Read \& write & 0 & 0.0 & 2 & 66.7 & 1 & 33.3 & 3 & 2.1 & $\mathrm{P}=0.529$ \\
\hline - Primary education & 0 & 0.0 & 4 & 80.0 & 1 & 20.0 & 5 & 3.6 & \\
\hline - Preparatory education & 0 & 0.0 & 9 & 90.0 & 1 & 10.0 & 10 & 7.1 & \\
\hline - Secondary education & 5 & 7.2 & 61 & 88.4 & 3 & 4.3 & 69 & 49.3 & \\
\hline - University education & 1 & 2.5 & 33 & 82.5 & 6 & 15.0 & 40 & 28.6 & \\
\hline \multicolumn{10}{|l|}{ Mother's occupation } \\
\hline - Working & 5 & 7.6 & 52 & 78.8 & 9 & 13.6 & 66 & 47.1 & $x^{2}=5503$ \\
\hline - Not working & 1 & 1.4 & 68 & 91.9 & 5 & 6.8 & 74 & 52.9 & $\mathrm{P}=0.064$ \\
\hline \multicolumn{10}{|c|}{ Father's level of education } \\
\hline - Illiterate & 0 & 0.0 & 4 & 80.0 & 1 & 20.0 & 5 & 3.6 & $\mathrm{X}^{2}=4.377$ \\
\hline - Read \& write & 0 & 0.0 & 1 & 100.0 & 0 & 0.0 & 1 & 0.7 & $\mathrm{P}=0.929$ \\
\hline - Primary education & 0 & 0.0 & 1 & 100.0 & 0 & 0.0 & 1 & 0.7 & \\
\hline - Preparatory education & 0 & 0.0 & 12 & 80.0 & 3 & 20.0 & 15 & 10.7 & \\
\hline - Secondary education & 3 & 6.5 & 40 & 87.0 & 3 & 6.5 & 46 & 32.9 & \\
\hline - University education & 3 & 4.2 & 62 & 86.1 & 7 & 9.7 & 72 & 51.4 & \\
\hline \multicolumn{10}{|l|}{ Father's occupation } \\
\hline - Working & 6 & 4.3 & 118 & 85.5 & 14 & 10.1 & 138 & 91.4 & $X^{2}=0.338$ \\
\hline - Not working & 0 & 0.0 & 2 & 100.0 & 0 & 0.0 & 2 & 8.6 & \\
\hline
\end{tabular}

Figure (V):.Conveys Distribution of the Study Group according To Overall Score of Knowledge by Department after the Educational Intervention.

It can be observed from the figure that more than half $(59.2 \%)$ of the study group in the welding department had good knowledge, whereas more than one third (34.3\%) of them had poor knowledge and only $6.5 \%$ of them had fair knowledge. Pertaining to lathing department, more than three quarters $(83.3 \%)$ of the students had good knowledge and more than one tenth $(16.7 \%)$ had poor knowledge. Also it can be noticed that more than half $(59.1 \%)$ of the students in foundry department had good knowledge, whereas more than one quarter $(31.8 \%)$ of them had fair knowledge and the minority $(9.1 \%)$ had poor knowledge. 


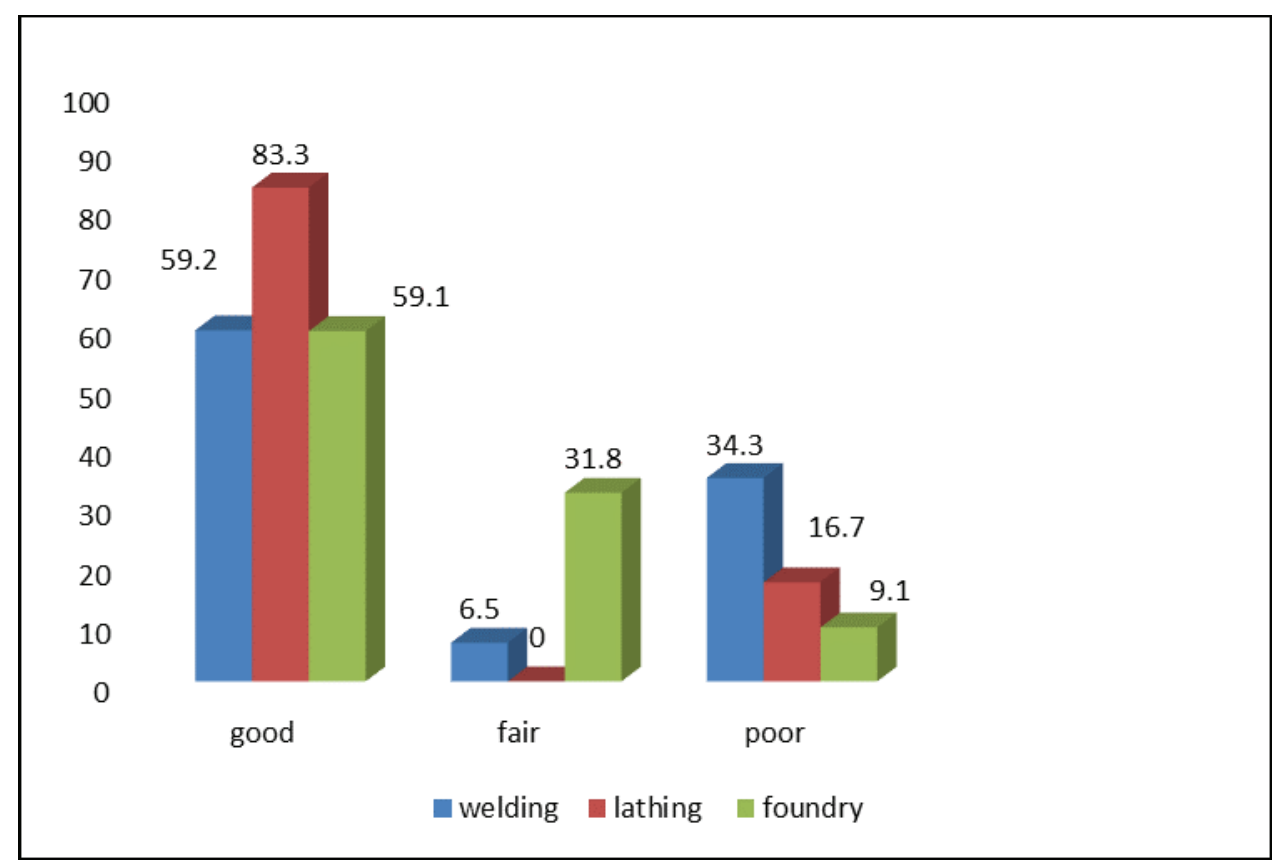

Table (VI): Available environmental safety measures in different mechanical workshops according to the scoring used in this study.

It was apparent that all observed workshops had incomplete safety measures in terms of housekeeping, ventilation, noise, lighting, machine safe working, manual tool, electricity, fire protection, first aid facility, and communication facility.

Table (VII): Available Environmental Safety Measures In Different Mechanical Workshops

\begin{tabular}{|c|c|c|c|c|c|c|c|c|c|c|c|c|}
\hline \multirow{3}{*}{ Items } & \multicolumn{6}{|c|}{ Pre program } & \multicolumn{6}{|c|}{ Post program } \\
\hline & \multicolumn{2}{|c|}{$\begin{array}{c}\text { Welding } \\
(\mathrm{n}=\mathbf{3})\end{array}$} & \multicolumn{2}{|c|}{$\begin{array}{c}\text { Lathing } \\
(n=4)\end{array}$} & \multicolumn{2}{|c|}{$\begin{array}{c}\text { Foundry } \\
(n=2)\end{array}$} & \multicolumn{2}{|c|}{$\begin{array}{c}\text { Welding } \\
(\mathrm{n}=3)\end{array}$} & \multicolumn{2}{|c|}{$\begin{array}{c}\text { Lathing } \\
(n=4)\end{array}$} & \multicolumn{2}{|c|}{$\begin{array}{c}\text { Foundry } \\
(n=2)\end{array}$} \\
\hline & No. & $\%$ & No. & $\%$ & No. & $\%$ & No. & $\%$ & No. & $\%$ & No. & $\%$ \\
\hline $\begin{array}{l}\text { House keeping } \\
\text { Incomplete } \\
\text { Complete } \\
\end{array}$ & $\begin{array}{l}3 \\
0\end{array}$ & $\begin{array}{c}100.0 \\
0.0\end{array}$ & $\begin{array}{l}4 \\
0\end{array}$ & $\begin{array}{c}100.0 \\
0.0\end{array}$ & $\begin{array}{l}2 \\
0 \\
\end{array}$ & $\begin{array}{c}100.0 \\
0.0\end{array}$ & $\begin{array}{l}1 \\
2 \\
\end{array}$ & $\begin{array}{l}33.3 \\
66.7 \\
\end{array}$ & $\begin{array}{l}1 \\
3 \\
\end{array}$ & $\begin{array}{l}25 \\
75\end{array}$ & $\begin{array}{l}0 \\
2\end{array}$ & $\begin{array}{l}0.0 \\
100\end{array}$ \\
\hline $\begin{array}{l}\text { Ventilation } \\
\text { Incomplete } \\
\text { Complete } \\
\end{array}$ & $\begin{array}{l}3 \\
0\end{array}$ & $\begin{array}{c}100.0 \\
0.0\end{array}$ & $\begin{array}{l}4 \\
0\end{array}$ & $\begin{array}{c}100.0 \\
0.0\end{array}$ & $\begin{array}{l}2 \\
0\end{array}$ & $\begin{array}{c}100.0 \\
0.0\end{array}$ & $\begin{array}{l}3 \\
0\end{array}$ & $\begin{array}{c}100 \\
0\end{array}$ & $\begin{array}{l}4 \\
0\end{array}$ & $\begin{array}{c}100 \\
0\end{array}$ & $\begin{array}{l}2 \\
0\end{array}$ & $\begin{array}{c}100 \\
0\end{array}$ \\
\hline $\begin{array}{l}\text { Noise } \\
\text { Incomplete } \\
\text { Complete } \\
\end{array}$ & $\begin{array}{l}3 \\
0 \\
\end{array}$ & $\begin{array}{c}100.0 \\
0.0 \\
\end{array}$ & $\begin{array}{l}4 \\
0 \\
\end{array}$ & $\begin{array}{c}100.0 \\
0.0 \\
\end{array}$ & $\begin{array}{l}2 \\
0 \\
\end{array}$ & $\begin{array}{c}100.0 \\
0.0 \\
\end{array}$ & $\begin{array}{l}3 \\
0 \\
\end{array}$ & $\begin{array}{c}100.0 \\
0.0 \\
\end{array}$ & $\begin{array}{l}4 \\
0 \\
\end{array}$ & $\begin{array}{c}100.0 \\
0.0 \\
\end{array}$ & $\begin{array}{l}2 \\
0 \\
\end{array}$ & $\begin{array}{c}100.0 \\
0.0 \\
\end{array}$ \\
\hline $\begin{array}{l}\text { Lighting } \\
\text { Incomplete } \\
\text { Complete }\end{array}$ & $\begin{array}{l}3 \\
0\end{array}$ & $\begin{array}{c}100.0 \\
0.0\end{array}$ & $\begin{array}{l}4 \\
0\end{array}$ & $\begin{array}{c}100.0 \\
0.0\end{array}$ & $\begin{array}{l}2 \\
0\end{array}$ & $\begin{array}{c}100.0 \\
0.0\end{array}$ & $\begin{array}{l}3 \\
0\end{array}$ & $\begin{array}{c}100.0 \\
0.0\end{array}$ & $\begin{array}{l}3 \\
1\end{array}$ & $\begin{array}{l}75.0 \\
25.0\end{array}$ & $\begin{array}{l}2 \\
0\end{array}$ & $\begin{array}{c}100.0 \\
0.0\end{array}$ \\
\hline $\begin{array}{l}\text { Machine safe working } \\
\text { Incomplete } \\
\text { Complete }\end{array}$ & $\begin{array}{l}3 \\
0\end{array}$ & $\begin{array}{c}100.0 \\
0.0\end{array}$ & $\begin{array}{l}4 \\
0\end{array}$ & $\begin{array}{c}100.0 \\
0.0\end{array}$ & $\begin{array}{l}2 \\
0\end{array}$ & $\begin{array}{c}100.0 \\
0.0\end{array}$ & $\begin{array}{l}3 \\
0\end{array}$ & $\begin{array}{c}100.0 \\
0.0\end{array}$ & $\begin{array}{l}4 \\
0\end{array}$ & $\begin{array}{c}100.0 \\
0.0\end{array}$ & $\begin{array}{l}2 \\
0\end{array}$ & $\begin{array}{c}100.0 \\
0.0\end{array}$ \\
\hline $\begin{array}{l}\text { Manual tool } \\
\text { Incomplete } \\
\text { Complete }\end{array}$ & $\begin{array}{l}3 \\
0\end{array}$ & $\begin{array}{c}100.0 \\
0.0\end{array}$ & $\begin{array}{l}4 \\
0\end{array}$ & $\begin{array}{c}100.0 \\
0.0\end{array}$ & $\begin{array}{l}2 \\
0\end{array}$ & $\begin{array}{c}100.0 \\
0.0\end{array}$ & $\begin{array}{l}3 \\
0\end{array}$ & $\begin{array}{c}100 \\
0\end{array}$ & $\begin{array}{l}4 \\
0\end{array}$ & $\begin{array}{c}100 \\
0\end{array}$ & $\begin{array}{l}2 \\
0\end{array}$ & $\begin{array}{c}100 \\
0\end{array}$ \\
\hline $\begin{array}{l}\text { Electricity } \\
\text { Incomplete } \\
\text { Complete }\end{array}$ & $\begin{array}{l}0 \\
3\end{array}$ & $\begin{array}{c}0.0 \\
100.0\end{array}$ & $\begin{array}{l}0 \\
4\end{array}$ & $\begin{array}{c}0.0 \\
100.0\end{array}$ & $\begin{array}{l}0 \\
2\end{array}$ & $\begin{array}{c}0.0 \\
100.0\end{array}$ & $\begin{array}{l}0 \\
3\end{array}$ & $\begin{array}{c}0.0 \\
100.0\end{array}$ & $\begin{array}{l}0 \\
4\end{array}$ & $\begin{array}{c}0.0 \\
100.0\end{array}$ & $\begin{array}{l}0 \\
2\end{array}$ & $\begin{array}{c}0.0 \\
100.0\end{array}$ \\
\hline $\begin{array}{l}\text { Fire protection } \\
\text { Incomplete } \\
\text { Complete }\end{array}$ & $\begin{array}{l}3 \\
0\end{array}$ & $\begin{array}{c}100.0 \\
0.0\end{array}$ & $\begin{array}{l}4 \\
0\end{array}$ & $\begin{array}{c}100.0 \\
0.0\end{array}$ & $\begin{array}{l}2 \\
0\end{array}$ & $\begin{array}{c}100.0 \\
0.0\end{array}$ & $\begin{array}{l}3 \\
0\end{array}$ & $\begin{array}{c}100.0 \\
0.0\end{array}$ & $\begin{array}{l}4 \\
0\end{array}$ & $\begin{array}{c}100.0 \\
0.0\end{array}$ & $\begin{array}{l}2 \\
0\end{array}$ & $\begin{array}{c}100.0 \\
0.0\end{array}$ \\
\hline $\begin{array}{l}\text { First aid facility } \\
\text { Incomplete } \\
\text { Complete }\end{array}$ & $\begin{array}{l}3 \\
0\end{array}$ & $\begin{array}{c}100.0 \\
0.0\end{array}$ & $\begin{array}{l}4 \\
0\end{array}$ & $\begin{array}{c}100.0 \\
0.0\end{array}$ & $\begin{array}{l}2 \\
0\end{array}$ & $\begin{array}{c}100.0 \\
0.0\end{array}$ & $\begin{array}{l}3 \\
0\end{array}$ & $\begin{array}{c}100.0 \\
0.0\end{array}$ & $\begin{array}{l}4 \\
0\end{array}$ & $\begin{array}{c}100.0 \\
0.0\end{array}$ & $\begin{array}{l}2 \\
0\end{array}$ & $\begin{array}{c}100.0 \\
0.0\end{array}$ \\
\hline
\end{tabular}




\begin{tabular}{|c|c|c|c|c|c|c|c|c|c|c|c|c|}
\hline \multirow{3}{*}{ Items } & \multicolumn{6}{|c|}{ Pre program } & \multicolumn{6}{|c|}{ Post program } \\
\hline & \multicolumn{2}{|c|}{$\begin{array}{c}\text { Welding } \\
(\mathrm{n}=3)\end{array}$} & \multicolumn{2}{|c|}{$\begin{array}{c}\text { Lathing } \\
(\mathrm{n}=4)\end{array}$} & \multicolumn{2}{|c|}{$\begin{array}{c}\text { Foundry } \\
(n=2)\end{array}$} & \multicolumn{2}{|c|}{$\begin{array}{l}\text { Welding } \\
(\mathrm{n}=3)\end{array}$} & \multicolumn{2}{|c|}{$\begin{array}{c}\text { Lathing } \\
(n=4)\end{array}$} & \multicolumn{2}{|c|}{$\begin{array}{c}\text { Foundry } \\
(n=2)\end{array}$} \\
\hline & No. & $\%$ & No. & $\%$ & No. & $\%$ & No. & $\%$ & No. & $\%$ & No. & $\%$ \\
\hline Communication facility & & & & & & & & & & & & \\
\hline Incomplete & 3 & 100.0 & 4 & 100.0 & 2 & 100.0 & 3 & 100.0 & 4 & 100.0 & 2 & 100.0 \\
\hline Complete & 0 & 0.0 & 0 & 0.0 & 0 & 0.0 & 0 & 0.0 & 0 & 0.0 & 0 & 0.0 \\
\hline
\end{tabular}

Table (IX): Safety Measures Adopted In Different Workshops During Work Process. It can be observed from the table that none of the welding workshops adopted complete safety measures during working process neither before nor after the educational intervention. On the contrary, complete safety measures were adopted in both lathing and foundry workshops in the pretest and posttest phases.

Table (IX): Safety Measures Adopted In Different Workshops during Work Process

\begin{tabular}{|c|c|c|c|c|c|c|c|c|c|c|c|c|}
\hline \multirow{3}{*}{ Items } & \multicolumn{6}{|c|}{ Pre program } & \multicolumn{6}{|c|}{ Post program } \\
\hline & \multicolumn{2}{|c|}{$\begin{array}{l}\text { Welding } \\
(\mathrm{n}=\mathbf{3})\end{array}$} & \multicolumn{2}{|c|}{$\begin{array}{c}\text { Lathing } \\
(n=4)\end{array}$} & \multicolumn{2}{|c|}{$\begin{array}{c}\text { Foundry } \\
(n=2)\end{array}$} & \multicolumn{2}{|c|}{$\begin{array}{c}\text { Welding } \\
(\mathrm{n}=\mathbf{3})\end{array}$} & \multicolumn{2}{|c|}{$\begin{array}{c}\text { Lathing } \\
(n=4)\end{array}$} & \multicolumn{2}{|c|}{$\begin{array}{c}\text { Foundry } \\
(n=2)\end{array}$} \\
\hline & No. & $\%$ & No. & $\%$ & No. & $\%$ & No. & $\%$ & No. & $\%$ & No. & $\%$ \\
\hline Welding safety & & & & & & & & & & & & \\
\hline Incomplete & 3 & 100.0 & - & - & - & - & 3 & 100.0 & - & - & - & - \\
\hline Complete & 0 & 0.0 & - & - & - & - & 0 & 0.0 & - & - & - & - \\
\hline Lathing safety & & & & & & & & & & & & \\
\hline Incomplete & - & - & 0 & 0.0 & - & - & - & - & 0 & 0.0 & - & - \\
\hline Complete & - & - & 4 & 100.0 & - & - & - & - & 4 & 100.0 & - & - \\
\hline Foundry safety & & & & & & & & & & & & \\
\hline Incomplete & - & - & - & - & 0 & 0.0 & - & - & - & - & 0 & 0.0 \\
\hline Complete & - & - & - & - & 2 & 100.0 & - & - & - & - & 2 & 100.0 \\
\hline
\end{tabular}

Table (X): Protective Devices in Different Mechanical Workshops. The table pointed out that body protection, feet protection, ear protection; hand protection, eye and face protection devices were not adopted in the all observed workshops before and after the educational intervention.

Table (X): Protective Devices in Different Mechanical Workshops

\begin{tabular}{|c|c|c|c|c|c|c|c|c|c|c|c|c|}
\hline \multirow{3}{*}{ Items } & \multicolumn{6}{|c|}{ Pre program } & \multicolumn{6}{|c|}{ Post program } \\
\hline & \multicolumn{2}{|c|}{$\begin{array}{c}\text { Welding } \\
(\mathrm{n}=3)\end{array}$} & \multicolumn{2}{|c|}{$\begin{array}{c}\text { Lathing } \\
(n=4)\end{array}$} & \multicolumn{2}{|c|}{$\begin{array}{c}\text { Foundry } \\
(n=2)\end{array}$} & \multicolumn{2}{|c|}{$\begin{array}{c}\text { Welding } \\
(\mathrm{n}=3)\end{array}$} & \multicolumn{2}{|c|}{$\begin{array}{c}\text { Lathing } \\
(n=4)\end{array}$} & \multicolumn{2}{|c|}{$\begin{array}{c}\text { Foundry } \\
(n=2)\end{array}$} \\
\hline & No. & $\%$ & No. & $\%$ & No. & $\%$ & No. & $\%$ & No. & $\%$ & No. & $\%$ \\
\hline \begin{tabular}{|l} 
Body protection \\
Incomplete \\
Complete \\
\end{tabular} & $\begin{array}{l}3 \\
0\end{array}$ & $\begin{array}{c}100.0 \\
0.0\end{array}$ & $\begin{array}{l}4 \\
0\end{array}$ & $\begin{array}{c}100.0 \\
0.0\end{array}$ & $\begin{array}{l}2 \\
0\end{array}$ & $\begin{array}{c}100.0 \\
0.0\end{array}$ & $\begin{array}{l}3 \\
0\end{array}$ & $\begin{array}{c}100.0 \\
0.0\end{array}$ & $\begin{array}{l}4 \\
0\end{array}$ & $\begin{array}{c}100.0 \\
0.0\end{array}$ & $\begin{array}{l}2 \\
0\end{array}$ & $\begin{array}{c}100.0 \\
0.0\end{array}$ \\
\hline \begin{tabular}{|c|} 
Feet protection \\
Incomplete \\
Complete \\
\end{tabular} & $\begin{array}{l}3 \\
0\end{array}$ & $\begin{array}{c}100.0 \\
0.0\end{array}$ & $\begin{array}{l}4 \\
0\end{array}$ & $\begin{array}{c}100.0 \\
0.0\end{array}$ & $\begin{array}{l}2 \\
0\end{array}$ & $\begin{array}{c}100.0 \\
0.0\end{array}$ & $\begin{array}{l}3 \\
0\end{array}$ & $\begin{array}{c}100.0 \\
0.0\end{array}$ & $\begin{array}{l}4 \\
0 \\
\end{array}$ & $\begin{array}{c}100.0 \\
0.0\end{array}$ & $\begin{array}{l}2 \\
0 \\
\end{array}$ & $\begin{array}{c}100.0 \\
0.0\end{array}$ \\
\hline \begin{tabular}{|l} 
Ear protection \\
Incomplete \\
Complete \\
\end{tabular} & $\begin{array}{l}3 \\
0\end{array}$ & $\begin{array}{c}100.0 \\
0.0\end{array}$ & $\begin{array}{l}4 \\
0\end{array}$ & $\begin{array}{c}100.0 \\
0.0\end{array}$ & $\begin{array}{l}2 \\
0\end{array}$ & $\begin{array}{c}100.0 \\
0.0\end{array}$ & $\begin{array}{l}3 \\
0\end{array}$ & $\begin{array}{c}100.0 \\
0.0\end{array}$ & $\begin{array}{l}4 \\
0\end{array}$ & $\begin{array}{c}100.0 \\
0.0\end{array}$ & $\begin{array}{l}2 \\
0\end{array}$ & $\begin{array}{c}100.0 \\
0.0\end{array}$ \\
\hline \begin{tabular}{|c|} 
Hand protection \\
Incomplete \\
Complete \\
\end{tabular} & $\begin{array}{l}3 \\
0 \\
\end{array}$ & $\begin{array}{c}100.0 \\
0.0 \\
\end{array}$ & $\begin{array}{l}4 \\
0 \\
\end{array}$ & $\begin{array}{c}100.0 \\
0.0 \\
\end{array}$ & $\begin{array}{l}2 \\
0 \\
\end{array}$ & $\begin{array}{c}100.0 \\
0.0 \\
\end{array}$ & $\begin{array}{l}3 \\
0 \\
\end{array}$ & $\begin{array}{c}100.0 \\
0.0\end{array}$ & $\begin{array}{l}4 \\
0 \\
\end{array}$ & $\begin{array}{c}100.0 \\
0.0 \\
\end{array}$ & $\begin{array}{l}2 \\
0 \\
\end{array}$ & $\begin{array}{c}100.0 \\
0.0\end{array}$ \\
\hline $\begin{array}{l}\text { Eye and face protection } \\
\text { Incomplete } \\
\text { Complete }\end{array}$ & $\begin{array}{l}3 \\
0\end{array}$ & $\begin{array}{c}100.0 \\
0.0\end{array}$ & $\begin{array}{l}4 \\
0\end{array}$ & $\begin{array}{c}100.0 \\
0.0\end{array}$ & $\begin{array}{l}2 \\
0\end{array}$ & $\begin{array}{c}100.0 \\
0.0\end{array}$ & $\begin{array}{l}3 \\
0\end{array}$ & $\begin{array}{c}100.0 \\
0.0\end{array}$ & $\begin{array}{l}4 \\
0\end{array}$ & $\begin{array}{c}100.0 \\
0.0\end{array}$ & $\begin{array}{l}2 \\
0\end{array}$ & $\begin{array}{c}100.0 \\
0.0\end{array}$ \\
\hline
\end{tabular}

\section{Discussion}

Adolescent population and their health are a very special issue and focus of attention globally for various reasons, as it is a period of rapid physical growth and emotional and psychological change. Health and quality of life during adulthood depend on health during adolescence ${ }^{(21)}$.

Safe work and workplace, for increased production and higher productivity, are necessary and hence promotion and protection of safe work and workplace are the complementary aspects of industrial development (22).

In technical schools, students have the highest injury rates, as they not only exposed to hazards from school 
environment, but also from workshops. Moreover, they spend nearly half of their time in workshop training, facing different types of actual and potential hazards ${ }^{(23)}$.

If students have to participate in activities to promote their health, they must be aware of the risks at work. Young workers (15-24 years old) have a disproportionately high rate of injuries in the workplace and many are employed on a casual, temporary or intermittent basis, in jobs that require low technical skill .One approach to reduce the burden of young worker injury is through improvements in the provision and effectiveness of safety training, initially at school and then in the workplace ${ }^{(24)}$.

The primary aim of any occupational health and safety program is the prevention of accidents and illness, which employs knowledge as the principal tool. Only accurate knowledge of the risks and adequate training in handling them can enable the worker to adopt appropriate behavior in a hazardous working environment ${ }^{(25)}$.

Previous studies concluded that technical education students had a limited knowledge of how to prevent health risks at work and lacked a systematic way to approach hazard control. Therefore, the aim of this study was to evaluate the effect of educational intervention about occupational safety on knowledge and practice of male students in technical mechanical secondary schools in El-Behira Governorate.

The current findings illustrated that less than half $(46.4 \%, 47.9 \%)$ of both the study and the control group respectively were aged between 16 to less than 17 years. While, a minority $(3.6 \%)$ of both groups were aged from 18 to less than 19 years. With a mean age of $16.39 \pm 0.71$ among the study group whereas among the control group $16.21 \pm 0.71$. Furthermore, less than two thirds $(63.6 \%)$ of the study group were living in urban areas compared to two thirds $(66.4 \%)$ of the control group.

Concerning the family income, the current study demonstrated that the vast majority of both groups (90\%) had not enough income to meet the demands of their lives, and the minority of both groups $(10 \%)$ had enough income. As regards to the crowding index, the present study demonstrated that the crowding index ranged from 1 to 10 persons/bed room among the study group and from 1-5 among the control group with a mean of (2.4+0.9) and $(2.1+0.6)$ respectively.

With respect to the relation between socioeconomic characteristics and level of knowledge, the present study illustrated that there was significant association between age of the students and their level of knowledge. On the other hand, no significant association was found between the level of knowledge and place of residence, crowding index, family income, parent's level of education and occupation.

Adolescents are considered to be a nutritionally vulnerable segment of the population. Due to enhanced growth during adolescence, the requirement of some minerals is of paramount important. A rapid growth rate combined with a marginal nutrient intake increases the risk of nutritional deficiencies in this population. Micronutrients such as iron and zinc are essential trace elements involved in the high growth rates of adolescents (26)

The nutritional status of adolescents contributes significantly to the health status of the community in which they reside. One of the major global health problems faced by developing countries today is under nutrition (27). The present study revealed that around two thirds of the study group (61.4\%) reported that they had three meals per day compared to more than two thirds (69.3\%) of the control group. This was consistent with the study conducted by Al-Isa A (2018) who found that nearly two thirds $(66 \%)$ of the studied sample had three meals daily ${ }^{(28)}$. Moreover, nearly two fifths $(41.4 \%)$ of the study group always had breakfast daily compared to more than half $(52.9 \%)$ of the control group. Furthermore, half of the study group (50\%) reported always intake of fast food compared to less than two third (63.6\%) of the control group.

Physical activity habits during adolescence and youth are likely to be important influences on habitual physical activity throughout adult life ${ }^{(28)}$.Opportunities for physical activity are largely determined by social, economic, and cultural factors as well as physical environments that influence access, availability and utilization ${ }^{(29)}$. The present findings illustrated that less than two thirds $(60.7 \%)$ of the study group didn't practice any type of exercise compared to more than three quarters $(83.6 \%)$ of the control group.

Sleep disorders in adolescents are common problems in families and can affect the social, emotional and educational performance ${ }^{(30)}$. The present study illustrated that the mean duration of nocturnal sleep was $5.75 \pm$ 1.68 for the study group and $5.59 \pm 1.40$ for the control group. This was contradicted with the study conducted by Reisi et al (2017) as the mean duration of nocturnal sleep was $8.38 \pm 1.17^{(31)}$. Moreover, the current findings revealed that nearly one tenth $(10.7 \%)$ of the study group had sleeping problems compared to less than half of the control group (48.6\%). These findings also lend credence to the research findings of Marques A et al (2019) (32)

Male students were significantly more likely than female students to engage in risk behavior in school ${ }^{(33)}$. Such behavior involving substance abuse, un-safe sex and irresponsible driving, may be seen as ways to prove their manliness ${ }^{(34)}$.

The results of the present study illustrated that more than one third (37.1) of the study group reported that they were smokers compared to about one fifth (20.7\%) of the control group. However, different picture was reported by (Nabil MM et al 2018) who indicated that more than one fourth $(26.5 \%)$ of the studied group were 
smokers ${ }^{(35)}$. Furthermore, the current findings demonstrated that neither of the study nor the control group was addicted to any drug. These results disagreed with the study conducted by (Boini S, et al 2017) who indicated that more than three quarters $(78.8 \%)$ of the studied sample were alcoholic and less than one tenth $(9.7 \%)$ were cannabis user ${ }^{(36)}$

Occupational safety and health should be the subject of education, as it prepares students for their future occupation, and further adult education, including retraining. Nowadays, as society supports technical education via dual learning, this issue is becoming particularly necessary. Schools ensure students' safety and health in education, training and other related activities as well as in the provision of school services ${ }^{(\mathbf{3 7})}$.

The present study portrayed that, before the implementation of the educational intervention, all the study group and the control one had poor knowledge regarding occupational health and safety in the school. This may be due to the limited access of information and there were no regular and periodic educational sessions about occupational hazards and occupational health and safety offered to them. In addition, there was a remarkable lack of clear policy, lack of investment and interest in occupational health and safety issues and a shortage of preventive strategies, implemented by the school managers and occupational health and safety supervisors which probably resulted in that significant decline in knowledge, before implementation of the program.

This finding is supported by (Tetemke et al 2014) who reported that the practice towards safety information is inadequate ${ }^{(38)}$. In addition, found that the workers have less knowledge level compared to other studies. Moreover, (Nasab et al 2009) depicted that knowledge, attitude and safe behaviors of the workers are unacceptable, so they suggested and concluded that managers should design and implement educational interventions to promote knowledge of workers ${ }^{(39)}$.

Although after the implementation of the educational intervention more than half $(52.8 \%)$ of the study group had good level of knowledge concerning occupational safety, whilst the entire control group had poor level of knowledge. There was highly significant impact of education on study's group's knowledge throughout the study. This remarkable and obvious improvement could be attributed to the effect of the implemented program. These findings strongly support the hypotheses that the level of workers' knowledge will be improved after implementation of the educational intervention.

Throughout the globe, most adults and many adolescents spend a great deal of their waking hours at work. The work provides a number of economic benefits. At the same time, people at work face a variety of hazards owing to chemicals, biological factors, physical agents, adverse ergonomic conditions, allergens, a complex network of safety hazards, and diversified psychosocial agents ${ }^{(40)}$.

The current findings depicted that the vast majority of the study and the control group had poor knowledge regarding occupational hazards before implementation of the educational intervention. This could be due to lack of periodic health education sessions about occupational hazards. These findings are congruent with other studies conducted by Shafik S 2016 and Gebrezgiabher et al $2019{ }^{(41,42)}$. However, these findings were contradicted with Adebola et al 2014 who stressed that the education and advice concerning specific work hazards are essential ${ }^{(43)}$.

While after the educational intervention, the results of the study revealed that, around two-fifths of the study group expressed higher total knowledge score compared to the control group. There was highly significant impact of education on study group knowledge throughout the study. This remarkable and obvious improvement could be attributed to the effect of the implemented program. This finding correspond with the results of (Padmini et al 2013) and (Sayapathi B 2014) ${ }^{(44,45)}$.

Every year, International Labor Organization (ILO) reports that, job related accidents and diseases claim estimated 2.34 million lives of men and women giving daily loss of 6,300 lives due to work related causes. Moreover, 160 million occupational diseases are reported each year (ILO 2013) ${ }^{(46)}$.

The current study elaborated that the vast majority of both the study and the control group had poor knowledge regarding school accidents before the implementation of the educational intervention. This finding may be explained by the lack of training programs. These findings are congruent with (Esaiyas et al 2018) and (Deebom et al 2019) ${ }^{(47,48)}$.

In point of fact, after implementation of the educational intervention, the study group showed significant improvement in their knowledge compared with the control group as less than half $(40 \%)$ of the study group had good knowledge regarding school accidents compared to only $2.9 \%$ of the control group. There was highly significant impact of education on study's group's knowledge across the three stages. This is in the same line with the results of other studies by (Saleh DA 2009) ${ }^{(49)}$

Safety is the prime requisite in all the work places. Workplace safety affects everyone in school workshop environment, including staff, students, and visitors. A safe and healthy working atmosphere is a basic necessity and has to be ensured in the technical education workshop without fail (Moksen 2013) ${ }^{(50)}$.

The current findings demonstrated that before the educational intervention, the entire study group and the control one had poor knowledge about safety measures that must be available in the workshop. This may be due to the limited access of information and there were no regular and periodic educational sessions about safety 
measures. These findings were in agreement with other studies conducted by (Andersson et al 2014) and (Balanay et al 2014) ${ }^{(20,51)}$

However, after the educational intervention, the current findings portrayed that the study group expressed higher total knowledge score compared to the control group as more than two thirds $(68.5 \%)$ of the study group had good level of knowledge concerning safety measures that must be available in the workshops whereas the entire control group still had poor level of knowledge. There was highly significant impact of education on study's group's knowledge throughout the study.

This remarkable and obvious improvement could be attributed to the effect of the implemented program, which included all information and skills required for the study group to help them to understand the necessity of safety measures that will help them to avoid the occurrence of higher levels of occupational hazards at the workplace and to the fact that it was custom-tailored to workers' needs. These findings strongly support the hypotheses "that the level of students' knowledge and practice will be improved after implementation of the educational intervention. These findings were comparable with other study conducted by (Gyekye et al 2015) ${ }^{(52)}$

In the general, school students have poor knowledge about safe working conditions during injuries, so most of them must be motivated to learn about first aid and basic life support which are components of chain survival for a person experiencing a life threatening injuries (Altintas 2015) ${ }^{(53)}$.

The present study demonstrated that all the study group and the vast majority of the control one $(98.6 \%)$ had poor level of knowledge regarding first aids before administration of the educational intervention. This finding may be explained by lack of training and health education sessions about first aid. These results were in agreement with (Dasgupta, et al 2014) and (Behairy et al 2015) ${ }^{(54,55)}$

However, the current findings revealed that after implementation of the educational intervention less than two thirds $(63.6 \%)$ of the study group had good level of knowledge concerning first aids, whereas the vast majority of the control group still had poor level of knowledge. There was highly significant impact of education on the study group's knowledge throughout the study. These findings were matching with the other studies conducted by (Al-Robaiaay 2013) and (Abd El-Hay et al 2015) ${ }^{(56,57)}$.

Students in industrial schools spend majority of the schooling day in the workshops for training. Hence, workshop environment must be taken into consideration to create a comfortable environment for students or teachers. Ventilation such as opened windows and adequate lighting is to be a great concern to ensure that the work is being carried out properly. Teachers also need to ensure adequate space for students to work in accordance with the number of students there. Safety means a condition resulting from a change in human behavior or the physical environment to reduce the likelihood of further hazards and reduce accidents. A large part of the safety compliance is the responsibility of all, acceptance of roles and awareness of the rules will be able to determine safe or otherwise of such an activity in order to ensure effective safety at the workshop teachers will need to identify to which extend the teachers and students practice safety management ${ }^{(\mathbf{5 8})}$.

The present study illustrated that all the observed workshops had incomplete environmental safety measures as regards to housekeeping, ventilation, noise, lighting, electricity, fire protection, manual tools, machine safe working, first aid facility and communication facility. This may be due to low financial resources allowed to technical mechanical secondary school workshops. Similar findings were reported by (Shafik $\mathbf{S}$ et al 2016) and (Deebom et al 2018) ${ }^{(48,59)}$. The study conducted by Shafik S et al (2016) illustrated that that half (50) of factories environment having good applicable of sanitation, ventilation and lighting while $100.0 \%$ had poor applicable for presence of safety measure and precaution sign. Deebom et al (2018) et al revealed that the workshops environment were restricted, had poor housekeeping, poor ventilation, poor illumination, the power tools were not properly grounded and insulated, had defective tools and equipment .

It was noticed in the present study that none of the welding workshops adopted complete safety measures during working process neither before nor after the educational intervention. These findings were in agreement with (Olagbegi et al 2013) and (Yekinni et al 2016) ${ }^{\mathbf{6 0 , 6 1 )}}$. The study carried out by Olagbegi et al (2013) portrayed that there were inadequate fire extinguisher in the laboratory; poor ventilation including hot shop and machine shop, faulty and old door locks, especially in the thermo fluid laboratory; little or no use of personal protective equipment, no working fire alarm, uncovered electrical panels and fuse boxes are related to causes of accidents in thermo fluid laboratory. Also, these findings corroborates with that of Yekinni (2016) who submitted that there were defective conditions of equipment; lack of signals and barricades; improper use of mechanical aid; power tools not properly grounded and insulated; floors, and inside passageway not kept clean.

Personal protective equipment are very effective in minimizing occupational injuries, accidents and other hazards which otherwise result in substantial human sufferings and financial losses. Personal Protective Equipments (PPEs) plays a prominent role in ensuring overall health and safety on the training workshops in industrial schools. As the students spend majority of the schooling day in the training workshop where they exposed to numerous physical, chemical and accidental hazards. Personal protective equipment includes face shields, safety glasses, hard hats, safety shoes, coveralls, gloves, ear protection, and respirators. Active cooperation and compliance of the workers are necessary for maximum benefits to be derived from PPE 
utilization $^{(62)}$

The current findings demonstrated that different means of personal protective equipment were not available in the different observed workshops. This might be due to financial resources shortage in these workshops and lack of awareness of the authorized persons about the necessity of personal protective equipment utilization. Otherwise, the current study is contradicted with the study conducted by (Sabitu K. et al 2009) who found that around one third $(34.2 \%)$ of the welders used one or more types of protective devices against workplace hazards with eye goggles $(60.9 \%)$, hand gloves $(50.3 \%)$ and boots $(34.5 \%)$ being more frequently used ${ }^{(63)}$.

\section{Conclusion}

Based upon the results of the present study, it could be concluded that:

Almost the entire study group had poor total knowledge, as well as poor total practice regarding occupational health and safety before application of the educational intervention. After implementation of the educational program a remarkable improvement was detected with highly statistical significant differences during pre-, post- and follow-up phases. Therefore, the educational program was successful in attaining its aim of positively changing knowledge, attitude and practice practices and of the studied group regarding occupational health and safety.

\section{Recommendation}

- Provision of personal protective equipment's for technical mechanical students in their training workshops.

- Conducting periodic health education sessions about the necessity of personal protective equipment utilization.

- It is necessary that first aid and basic life support courses must be a part of the curricula in industrial secondary school.

- The ministry of education should monitor technical mechanical workshops periodically.

- Conducting health education sessions about dangers of risk taking behaviors among male students in secondary schools.

- First aid and basic life support guidelines should be available to all students at school in order to decrease the early morbidity and mortality of accidents and emergencies

- It is recommended to carry out a regular health education session for the students to raise their knowledge in the occupational health and safety aspects.

\section{References}

1. World Health Organization (WHO).2015. Adolescent heath: mortality and burden of disease attributable to selected major risks. Geneva: WHO; [cited 16 October 2019]. Available from: https://www.who.int/maternal_child_adolescent/adolescence.

2. The National Agency for Education.2017. The Swedish National Agency for Education supervises and provides support for better schooling. Stockholm, Sweden: The National Agency for Education.

3. Moustafa EK.(2016). The Impact of Cultural Contex on The Egyptian Technical and Voactional Education and Training and Training System. Scientific Journal for Economy and Commerce;3:57-74.

4. Sofoluwe AO.(2013). Re-engineering vocational and technical education (VTE) for sustainable development in North Central Geo-Political Zone, Nigeria. Educational Research and Reviews.8(19):1842-9.

5. Paul B. (2014). Improving the quality and shape of technical education and training: Is it a low-level training or a tool to achieve comprehensive and sustansial development? Prospectives;44:291-311.

6. Schmidt C (2010). Vocational education and training (VET) for youths with low levels of qualification in Germany. Education \& Training. 52(5):381-90.

7. Zidan SR.(2016).A Field study of the performance of the managers of technical industrial schools in Elfayoum governorate. Faculty of Education Journal.;6(2):390-424.

8. Chen SC.(2010). The current status of general health education curriculum in technical institutes and universities in Taiwan. Creative Education;1(1):62-7.

9. Cohen H, Colligan MJ. (2013). Assessing Occupational Safety and Health Training: A literature review. DHHS (NIOSH) Pub. Number 98-145. Cincinnati, OH: National Institute for Occupational Safety and Health.

10. Burke MJ, Holman D, Birdi K.(2014) A walk on the safe side: The implications of learning theory for developing effective safety \& health training. In: Hodgkinson GP, Ford JK, (eds). International review of industrial and organizational psychology. London: Wiley; 1-41.

11. National Institute of Occupational Safety and Health, Data Overview of Young Worker Deaths and Injuries, United States 2011.Available at http://www.cdc.gov/ niosh/topics/youth/chtpkgfig5.html. Accessed on 8 May 2019. 
12. Friend M, Kohn J. (2014). Fundamentals of Occupational Safety and Health. $6^{\text {th }}$ ed. USA: Bernan Press; 2-3.

13. Adebola JO.(2014). Knowledge, attitude and compliance with occupational health and safety practices among pipeline products and marketing company (PPMC) staff in Lagos. Merit research journal of medicine and medical sciences;2(8):158-73.

14. Guzys, D., \&Petrie, E.(2014).An Introduction to Community and Primary Health Care.1st ed., U.S.A: Cambridge University Press; p.323. ISBN: $1107513529,9781107513525$.

15. Shendell DG, Noomnual S, Plascak J, Apostolico AA. (2018). Injuries among young workers in careertechnical-vocational education and associations with per pupil spending. BMC public health;18(1):1190.

16. Apostolico AA, Shendell DG.(2016).Injury surveillance and associations with socioeconomic status indicators among youth/young workers in New Jersey secondary schools. Environmental health;15:22.

17. Cooper K, Gosnell K.(2015). Foundations and Adult Health Nursing. $7^{\text {th }}$ ed. Canada: Elsevier Health Sciences; 2055.

18. Friend, M.\& Kohn, J. (2010). Fundamentals of occupational safety and health. 5th ed., U.S.A: Government Institute an imprint of the scarecrow Press, Inc; 177- 178.

19. Gebrezgiabher B, Tetemke D, Yetum T. (2013). Awareness of Occupational Hazards and Utilization of Safety Measures among Welders in Aksum and Adwa Towns, Tigray Region, Ethiopia,Journal of environmental and public health. 2019; 2019:4174085.

20. Andersson IM, Gunnarsson K, Rosèn G, Moström Åberg M.(2014). Knowledge and Experiences of Risks among Pupils in Vocational Education. Safety and health at work;5(3):140-6.

21. Bassiouny El-Lassy R , Abd El-Aziz Madian A. (2013). Healthy lifestyle in adolescence- Issues and Challenges for the Egyptian Health Sector. 4th international nursing conference, Oman, 1-22.

22. U. Upadhyaya. (2013).Occupational health, safety and environment in the construction sector," in Issue of the World of Work in Nepal, General Federation of Nepalese Trade Unions, Kathmandu, Nepal.

23. Guidotti, T. (2011).Global Occupational Health. 1st ed., New York, U.S.A: Oxford University Press; p p. 3 5 \& 15-16 .ISBN 978-0-19-538000-2.

24. Thamrin Y, Pisaniello D and Stewart S (2010): Time trends and predictive factors for safety perceptions among incoming South Australian university students. Journal of Safety Research; 41: 59-63.

25. Magoro, F. (2012). Knowledge, Attitude and Practices Regarding Personal Protective Equipment Amongst Stevens Lumber Mills employees in the Capricorn district of Limpopo Province, South Africa. A mini dissertation submitted in partial fulfillment of the requirements for the degree of master of public, in the school of Health Science, Faculty of Health Science, University of Limpopo.P8

26. Kabir Y, Mohammad H, Saleh F, Obaid W.(2010). Dietary pattern, nutritional status, anaemia and anaemiarelated knowledge in urban adolescent college girls of Bangladesh. J Pak Med Assoc;60 (8).

27. Mushtaq MU, Gull S, Khurshid U, Shahid U, Shad MA, et al. (2011) Prevalence and socio-demographic correlates of stunting and thinness among pakistani primary school children. BMC Public Health 11: 790.

28. Leslie E et al.(2012).University campus settings and the promotion of physical activity in young adults: lessons from research in Australia and the USA. Health Education, 101(3):116-125.

29. Ziglio E. Addressing the socioeconomic determinant of healthy eating habits and physical activity levels among adolescents (Foreword). (2013).Copenhagen, World Health Organization Regional Office for Europe (www.euro.who.int/ data/assets/pdf file/0005/98231/e89375.pdf, accessed 30 June 2011.

30. Dimitriou D, Le Cornu Knight F, Milton P.(2015). The Role of Environmental Factors on Sleep Patterns and School Performance in Adolescents. Front Psychol; 6: 1717.

31. Reisi M, Jalilian R, Azizi Gh, Rashti A, Faghihi nia J, Akbari M, et al. (2017). Academic Performance, Sleep Disorders and Their Association in Middle School Students in Iran. Int J Pediatr; 5(3): 4541-49. DOI: 10.22038/ijp.2017.21374.1794.

32. Marques A, Demetriou Y, Tesler R, Gouveia ER, Peralta M, Matos MG.(2019). Healthy Lifestyle in Children and Adolescents and Its Association with Subjective Health Complaints: Findings from 37 Countries and Regions from the HBSC Study. International journal of environmental research and public health.;16(18):E3292.

33. SINGH SUNITHA and GOPALKRISHNA GURURAJ.(2014). Health behaviors \& problems among young people in India: Cause for concern \& call for action Indian J. Med. Res., 140, August, pp. 185-208.

34. FOX H., McMANUS M, and ARNOLD K.: Significant Multiple Risk Behaviors among U.S. High School Stu-dents, National Alliance to Advance Adolescent Health; Fact Sheet, No. 8, 2015.

35. Nabil MM, Sadek M, Magdy H.(2018).Common Health Problems and Risk Behaviors among Adolescent Males in Governmental Secondary Schools, Assuit Governorate. The Medical Journal of Cairo University;86(1):137-44.

36. Boini S, Colin R, Grzebyk M.(2017). Effect of occupational safety and health education received during schooling on the incidence of workplace injuries in the first 2 years of occupational life: a prospective study. BMJ open.;7(7):e015100. 
37. Raykov, M., \& Taylor, A. (2013). Health and safety for Canadian youth in trades. Just Labour20, 33-50.

38. Tetemke, D., Alemu, K., Tefera, Y., \& et al. (2014). Knowledge and practices regarding safety information among textile workers in Adwa town, Ethiopia. Science Postprint1(1): 1-5.e00015. doi:10.14340/spp.2014.01A0004.

39. Nasab, H., Tavakoli, R., \& Ghofranipour, F. (2009). Evaluation of Knowledge, Attitude and Behavior of Workers towards Occupational Health and Safety. Iranian Journal of Public Health. 38(2):125-129.

40. Nickitas, M., Middaugh, D., \& Aries, N. (2011). Policy and Politics for nurses and other health professional. New York, U.S.A: Jones and Bartlett publishers. LLC; P. 40.

41. Shafik S, El-Mohsen A.(2012). Occupational health: health promotion program to improve health workers in Tourah cement factory. The Journal of American Science;8(3):486-96.

42. Gebrezgiabher B, Tetemke D, Yetum T.(2019). Awareness of Occupational Hazards and Utilization of Safety Measures among Welders in Aksum and Adwa Towns, Tigray Region, Ethiopia, 2013. Journal of environmental and public health;8(1):46-51.

43. Adebola JO.(2014). Knowledge, attitude and compliance with occupational health and safety practices among pipeline products and marketing company (PPMC) staff in Lagos. Merit research journal of medicine and medical sciences;2(8):158-73.

44. Padmini, D., \& Venmathi, A. (2013). Creating Awareness on Occupational Health and Safety among Workers Employed in Garment Industries. Ijsr - International Journal of Scientific Research; 2 (12 ).275277. ISSN No $2277-8179$.

45. Sayapathi, B., Su, A., \& Koh, D. (2014). Knowledge, Attitudes and Practice in Relation to Noise-Induced Hearing Loss in Two Factories, Malaysia.Research Journal of Biological Sciences.9(6): 197-204.ISSN: 1815-8846.

46. ILO.The prevention of occupational diseases. International Labor Organization, Geneva; 2013.

47. Esaiyas et al . (2018). Occupational Health and Safety Related Knowledge, Attitude and Practice among Wood and Metal Workers in Hawassa, Ethiopia. ARRB, 22(6): 1-9 .Article no.ARRB.38958.

48. Deebom MT, Ojobah LO.(2019). Implementation of Safety Practices for Enhancing Quality in Instructional Delivery in Electrical/Electronic Workshops in Rivers State Technical Colleges. International Journal of Latest Research in Humanities and Social Science;1(1):26-33.

49. Saleh DA, Elghorory LM, Shafik MR and Elsherbini EE (2009): Improvement of Knowledge, Attitudes and Practices of Health Care Workers Towards the Transmission of Blood-Borne Pathogens: An Intervention Study J Egypt Public Health Assoc; 84 (5-6):423-41.

50. Moksen A.(2013). Workshop Safety Management Amongst Students and Teachers at Vocational Secondary Schools. Journal of Resources Development and Management; (4): 41-53 doi:166756107.

51. Balanay JA, Adesina A, Kearney GD, Richards SL.(2014). Assessment of occupational health and safety hazard exposures among working college students. American journal of industrial medicine;57(1):114-24.

52. Gyekye S, Salminen S (2015). Responsibility Assignment at the workplace: A Finnish and Ghanaian Perspective. Blackwell Synergy.

53. Altintas K.H., Aslan D., Yidiz A.N., Subasi N., Elcin M., Odabasi O., Bilir N. and Sayek T.,.(2015). the Evaluation of First Aid and Basic Life Support Training for the First Year University Students, Tohoku J. Exp. Med.; (205): pp 157-169.

54. Dasgupta A., Bandyopadhyay L. and Das M.(2014). Effectiveness of Health Education in Terms of Knowledge Acquisition on First- Aid Measures among School Students of a Rural Area of West Bengal, Med. Res. Chron; 1 (2): 84-91.

55. Behairy AS, Al-Batanony MA. (2015). Effectiveness of First-Aid and Basic Life Support Intervention Program on School Health Advisors. Journal of Health, Medicine and Nursing-An International Peerreviewed Journal;24:136-44.

56. Al-Robaiaay Y. (2013). Knowledge of primary school teachers regarding first aid in Baghdad Al-Rusafa, Al-Kindy Col Med J; 9(1): 59.

57. Abd El-Hay SA, Ibrahim NA, Hassan LA.(2015). Effect of Training Program Regarding First Aid and Basic Life Support on the Management of Educational Risk injuries among Students in Industrial Secondary Schools. IOSR Journal of Nursing and Health Science;4(6):32-43.

58. Umunadi EK.(2011). Provision of Equipment and Facilities in Vocational and Technical Education for Improving Carrying Capacity of Nigeria's Tertiary Institution. In the Proceedings of the 1st International Technology, Education and Environment Conference. Held at Omoku-Nigeria.

59. Shafik S, Abd El-Aal E.(2016). Occupational Health Program on Preventing Hazard among Child Labor. IOSR Journal of Nursing a nd Health Science (IOSR-JNHS), 5 (3) :70-80.

60. Olagbegi, P. O., Kwasi, C. C. \& Ugbi, B. A. (2013). Assessment of Health and Safety Practice in Engineering workshop. International Journal of Engineering Science, 2(7), 297-301.

61. Yekinni, S. A. (2016). Methodological Needs of Electrical/Electronic Workshop Accident Prevention in 
Technical Colleges in South Western Part of Nigeria. Journal of Information Engineering and Applications, 6 (9): 44-52.

62. Johnson O, Motilewa O.(2016). Knowledge and Use of Personal Protective Equipment among Auto Technicians in Uyo, Nigeria. British Journal of Education, Society \& Behavioural Science.;15: 1-8. Doi:10.9734/BJESBS/2016/24546.

63. Sabitu K, Iliyasu Z, Dauda MM. (2009). Awareness of occupational hazards and utilization of safety measures among welders in Kaduna metropolis, northern Nigeria. Annals of African medicine;8(1):46-51. 